\title{
Exogenous attention generalizes perceptual learning in adults with amblyopia
}

\author{
Mariel Roberts ${ }^{1^{*} \dagger}$ \\ \& Marisa Carrasco ${ }^{1,2^{*}}$ \\ ${ }^{1}$ Department of Psychology, ${ }^{2}$ Center for Neural Science, \\ New York University, New York, New York 10003
}

\begin{tabular}{|c|c|c|}
\hline Authors & Mariel Roberts & Marisa Carrasco \\
\hline Address & $\begin{array}{c}\text { 6 Washington Place } \\
\text { New York, NY 10003 }\end{array}$ & $\begin{array}{c}\text { 6 Washington Place } \\
\text { New York, NY 10003 }\end{array}$ \\
\hline Phone number & $(212) 998-3894$ & $(212) 998-8328$ \\
\hline Email address & mariel.roberts@nyu.edu & marisa.carrasco@nyu.edu \\
\hline ORCID identifier & $0000-0003-1379-4172$ & $0000-0002-1002-9056$ \\
\hline
\end{tabular}

Keywords: perceptual learning, amblyopia, visual attention, neuroplasticity, contrast sensitivity, acuity, specificity, transfer

"co-corresponding authors

†lead contact 


\section{SUMMARY}

Visual perceptual learning (VPL), or improved performance after practicing the same visual task, is a behavioral manifestation of the impressive neuroplasticity in the adult brain. However, its practical effectiveness is limited because improvements are often specific to the trained conditions and require significant time and effort. Thus, it is critical to understand the conditions that promote learning and its transfer. Covert spatial attention helps overcome VPL location and feature specificity in neurotypical adults, but whether it can for people with atypical visual development is unknown. Here we show that involuntary attention helps generalize learning beyond trained spatial locations in adults with amblyopia, an ideal population for investigation given their asymmetrically developed, but highly plastic, visual cortex. Our findings provide insight into the mechanisms underlying changes in neuro(a)typical brain plasticity after practice. Further, they reveal that attention can enhance the effectiveness of perceptual learning during rehabilitation of visual disorders.

\section{INTRODUCTION}

Visual perceptual learning (VPL) refers to enhancements in perceptual sensitivity or discriminability after extensive practice (reviews[1-5]). There are significant translational implications for training visual expertise and rehabilitation of visual disorders. However, VPL improvements can be modest and are often characterized by specificity (reviews[1-5]). In most cases, performance improvements do not transfer beyond the trained retinal location and stimulus feature, and in some cases, they are specific to the trained eye. Thus, there is significant incentive to design training regimens that promote VPL and its transfer to untrained conditions. Elucidating the specific conditions that promote learning and generalization provides insight into the potential mechanisms and neural substrates underlying plasticity in the (a)typical adult brain. Protocols that require training with a second task, i.e., "piggybacking", a form of double training (e.g.[6,7]) and interleaving tasks [8], facilitate VPL to untrained locations (e.g.[6-8]) and features (e.g.[8]). However, these protocols increase the time and cognitive demands of training, which is particularly problematic for populations whose visual systems are already taxed, including adults with amblyopia.

Amblyopia is a neurodevelopmental disorder of the cortex characterized by interocular disparities [9-12]. Although typically considered a foveal disorder [13], its deficits and their neural markers are also present across the visual field [14-18]. Whether due to misalignment of the eyes (strabismic subtype), optical blur (anisometropic subtype), or a physical blockage of light (deprivation subtype), people with amblyopia rely on their stronger eye for most visual tasks while actively suppressing the processing of visual input to their weaker amblyopic eye. However, even their dominant "fellow" eye is impaired relative to those of neurotypical observers (review[19]). Despite wearing maximal optical correction, adults with amblyopia exhibit pronounced impairments in a diversity of tasks, e.g., contrast sensitivity, visual acuity, crowding, position discrimination, spatial interaction, letter recognition, stereopsis, visual search, counting, multiple-object tracking, and oculomotor and handeye coordination tasks (reviews[10,12,20-22]). Thus, the amblyopic brain is a useful model system for investigating neuroplasticity as well as its limits.

Visual training has been shown to reduce many of these deficits, specifically in contrast sensitivity, visual acuity, crowding, position discrimination, spatial interaction, letter recognition, and stereopsis (reviews[9,10,12,20-23]). Though not well understood, VPL is relatively widespread in amblyopic adults, often showing transfer to untrained stimulus features (e.g., spatial frequencies, 
orientations), visual dimensions (e.g., acuity, stereopsis), and to the fellow eye (reviews[9,12,2023]). After training the weaker eye, experimenters can measure differences in VPL improvement and transfer compared to the stronger fellow eye, which provides an informative within-subject "control" condition. In addition to their contribution to our understanding of neuro(a)typical brain functioning, findings from VPL studies with amblyopic patients can have translational implications for improving the efficiency of rehabilitation methods for visual disorders.

Spatial covert attention -the selective processing of visual information without accompanying eye movements- improves performance in a variety of detection, discrimination and localization tasks (reviews[24-26]). Recent studies manipulating observers' covert spatial attention during practice [27-31] have revealed that selective attention can enable VPL [27], and help overcome specificity [28-30] in neurotypical adults. Observers who trained with exogenous (involuntary) attention acquired VPL, but those who trained without focused attention under otherwise identical conditions did not [27]. Moreover, deploying exogenous [28] or endogenous (voluntary;[29]) spatial attention during training facilitates the transfer of improved orientation discrimination to untrained locations. Exogenous attention also facilitates VPL transfer in visual acuity to untrained stimulus locations and features [30]. In these studies, to isolate the attentional effect, separate groups of observers were trained with either valid attentional cues, i.e., which focused spatial attention at the target location, or neutral cues, i.e., which evenly spread spatial attention across all spatial locations. All groups were tested with neutral cues before and after training, such that evidence of VPL or transfer was only attributable to attentional allocation during training.

Given that spatial attention cues typically improve behavioral performance in amblyopia [16-18,32], and that attention helps overcome VPL location [28-30] and feature [30] specificity in neurotypical adults, here we investigated whether and to what extent exogenous attention facilitates VPL and its transfer in adults with amblyopia. There are alternative hypotheses for this open question. On the one hand, given that people with amblyopia benefit from attention in basic visual tasks as neurotypicals do [24-26], we could hypothesize that training with attention may also facilitate location and feature transfer. On the other hand, given underactivation in primary visual cortex (review[33]), atypical neural signaling across all stages of the visual hierarchy (e.g.[34,35], review[33]), and multiple anomalies in the responsivity and topography of neurons in the amblyopic brain (e.g.[13]; reviews[11,33,36]), we could hypothesize that attention may not interact with VPL in the amblyopic brain in a similar manner as in neurotypicals. Furthermore, given that amblyopic adults already show more generalized improvements than neurotypical adults, it is possible that attention would not be able to further potentiate VPL or its transfer to a measurable degree.

Two groups of adults with strabismic, anisometropic or mixed amblyopia (see Table 1 of Materials \& Methods) trained using Gabor stimuli set at individuals' contrast thresholds with their amblyopic eye on a two-alternative forced-choice (2-AFC) orientation discrimination task mediated by contrast sensitivity (Figure 1a; see Main task trial sequence under Materials \& Methods). The behavioral effects of both types of attention on this task, as well as their neural correlates, have been wellcharacterized in neurotypical observers (e.g.[37-46]), and their behavioral effects have been wellestablished for special populations (amblyopia[16], ASD[47-48], ADHD[49]). 


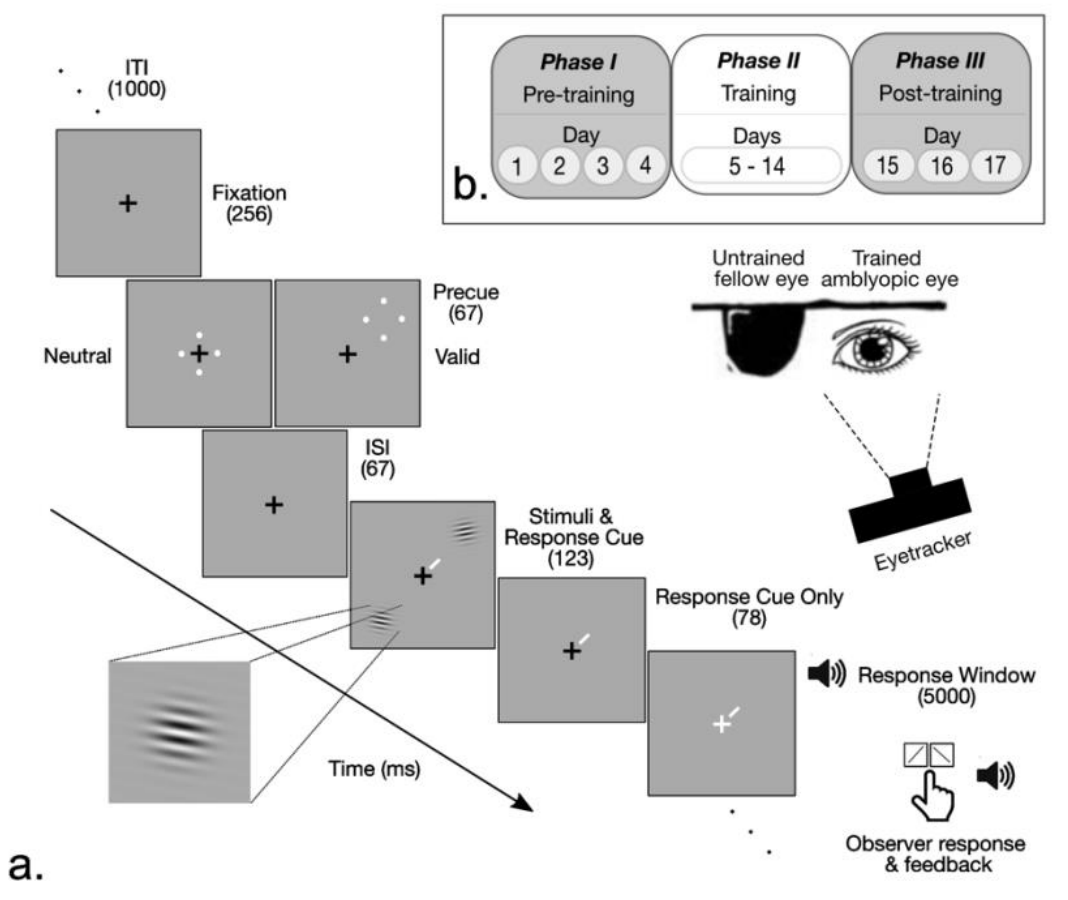

Figure 1. Experimental protocol. a. Trial sequence for the main training task. The size of the stimuli has been enlarged for improved visibility. b. Overview of study procedure.

To assess whether exogenous attention helps overcome location specificity, each group trained (Figure 1b) either with peripheral cues (Attention group) or central cues (Neutral group). In the days immediately preceding (Day 4 of the Pre-training phase; Figure 1b) and after (Day 15 of the Post-training phase; Figure 1b) the training sessions, we measured their performance on the same task in each eye at the trained and untrained diagonal meridians. In addition, to assess the degree of eye, feature and task transfer in both groups, we measured changes in some of the classic visual impairments of amblyopia -broadband foveal contrast sensitivity, visual acuity, crowding at the fovea and binocular stereoacuity- using a curated battery of tasks (see Supplemental Information for details).

\section{RESULTS}

Analysis. We measured changes in d' as the primary dependent variable to index changes in observers' perceptual sensitivity independent of their response criterion. d' was calculated as the zscore of the proportion of hits (arbitrarily chosen as when target was CCW, observer reported CCW) minus the z-score of the proportion of false alarms (target was CCW, observer reported CW; e.g.[17,41,43-46]). To avoid infinite values, we adopted the conservative log-linear rule in which 0.5 was added to the proportion of hits, misses, correct rejections and false alarms prior to computing d' [50]. To account for between-subject differences and compare learning across different tasks with different units of measurement, we conducted complementary analyses with our own performance measure -normalized proportion change- which we defined as the difference between post-test and pre-test performance divided by the sum of their values. A positive normalized proportion change indicated improvement. Thus, for those tasks in which a lower score is better (e.g., logMar visual acuity), the change was calculated as pre-test minus post-test performance. 
We designed our task to promote changes in perceptual sensitivity (d'). Observers were encouraged to be as accurate as possible. Median RTs for correct trials served as a secondary variable to assess potential speed-accuracy tradeoffs. RTs were measured relative to the simultaneous onset of the Gabor stimuli and response cue (Figure 1a), which remained onscreen for $123 \mathrm{~ms}$. The Gabors disappeared, leaving only the response cue, and observers had to wait an additional $78 \mathrm{~ms}$ before they were allowed to respond within a 5-s window, to further encourage observers to be as accurate rather than as fast as possible. In our experimental design, as in many RT tasks (e.g.[51]), it was impossible to distinguish the degree to which reaction time (RT) differences reflected changes in speed of processing, discriminability, response criterion, and/or motor learning (e.g.[49,52]).

We conducted all ANOVAs in R with ezANOVA using the Type-III sums of squares approach. We always included Observer as a random factor, Eye (amblyopic, fellow), Diagonal (trained, untrained) and Session (pre-test, post-test) as fixed within-subjects factors, and Group (Neutral, Attention) as a fixed between-subjects factor. Planned comparisons were two-tailed paired t-tests, and, when appropriate, Bonferroni correction was applied to correct for multiple comparisons. All reports of variability represent \pm 1 standard error of the mean.

Mean Contrast. We equated performance ( 1.3 d') between Eyes and Groups before training (Figure S1, top row) using an adaptive staircase procedure to estimate monocular contrast thresholds. A 2-way (Eye: amblyopic, fellow X Group: Neutral, Attention) mixed-design ANOVA of mean contrast threshold revealed a significant main effect of Eye $\left[F(1,18)=11.7, p=.003, \eta^{2} G\right.$ $=.215]$, but neither a significant main effect of Group $\left[F(1,18)=3.09, p=.096, \eta^{2} G=.091\right]$, nor a significant interaction $[F(1,18)<1]$. Both the Neutral and Attention groups required about twice the contrast in their weaker amblyopic eye $[M=35.0 \pm 5.16 \%]$ than the corresponding fellow eye $[M=17.0 \pm 2.98 \%]$ to obtain similar performance.

Pre-test and post-test performance on the main task. We first assessed whether and how exposure to a valid peripheral precue during training differentially affects the magnitude and specificity of learning. A 4-way mixed ANOVA on d' for all observers (see Supplemental Information) revealed that neither the main effect of Eye (amblyopic, fellow) nor its interaction with the other factors of Session (pre-test, post-test), Diagonal (trained, untrained) and Group (Neutral, Attention) were significant (all $p>.3$; Figure S1, top row). Thus, we collapsed across the amblyopic and fellow eye data for the following analyses.

Figure 2 plots the post-test versus pre-test performance (top row: d', bottom row: RT) collapsed across both eyes of individual observers at the trained (left column) and untrained (middle column) diagonals, as well as the normalized proportion change in performance at one diagonal relative to the other (right column). Training improved performance for most observers at the trained diagonal (Learners; filled shapes), but three observers in each group did not improve (Non-Learners; unfilled shapes). A greater proportion of the Learners (i.e., observers who exhibited a positive normalized proportion change in d' at the trained diagonal of the main training task) exhibited transfer to the untrained diagonal in the Attention group $(6 / 6,100 \%)$ than in the Neutral group $(3 / 7,43 \%)$. Further, the normalized proportion change in d' was more often similar at the trained and untrained diagonals for the Attention group than the Neutral group, as shown by the majority of green shapes clustered near the unity line compared to the majority of blue shapes clustered near the horizontal line in the right column of Figure 2. 


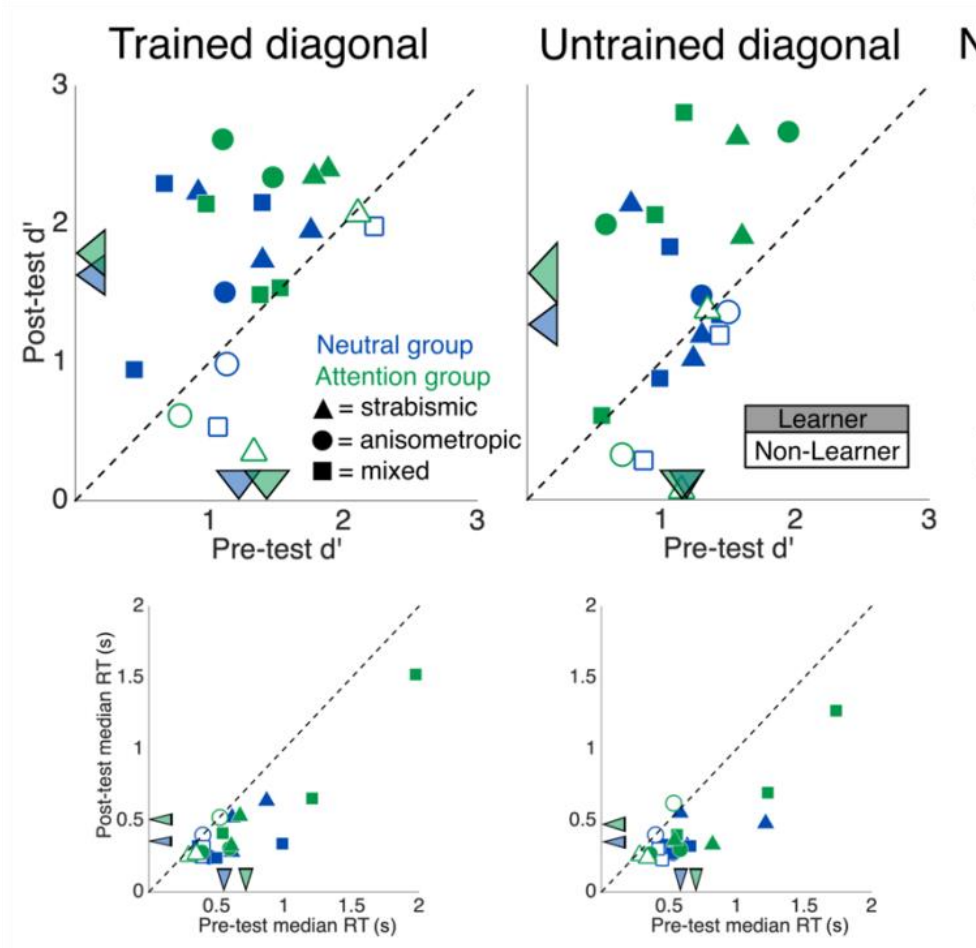

Normalized proportion change
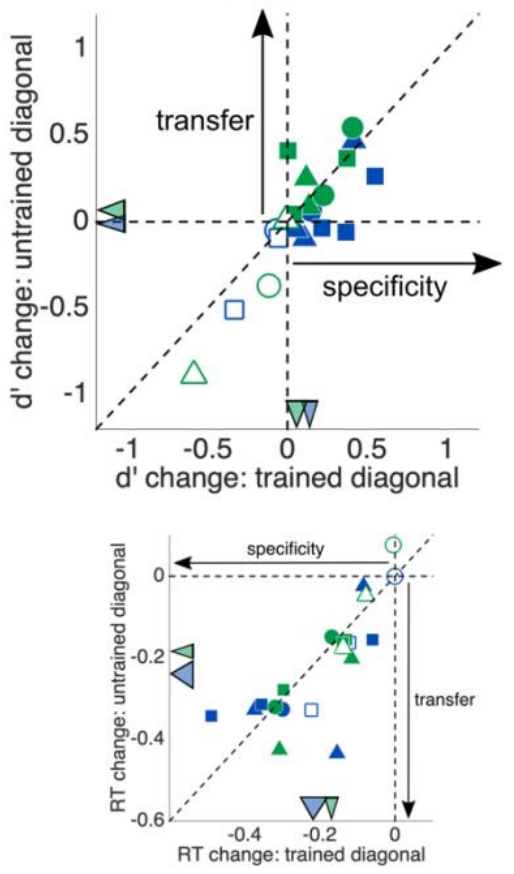

Figure 2. Performance (top row: d', bottom row: RT) collapsed across both eyes of 20 individual observers on the training task at post-test versus pre-test at the trained diagonal (left column), untrained diagonal (middle column), and the relative normalized proportion change at each diagonal (right column; data to the right of the vertical line indicate improvement at the trained diagonal; data above the horizontal line but below the unity line indicate partial transfer; data along the unity line indicate complete transfer; data above the unity line reveal greater improvement at the untrained compared to the trained diagonal). The colored arrows pointing to the axes indicate group means, and their widths represent \pm 1 within-groups SEM for each condition [53]. See also Figures S1, S8, and S9.

RTs were faster for most observers at post-test than pre-test for both the trained diagonal (left column) and untrained diagonal (middle column). Overall, the benefit was similar at both the trained and untrained diagonals for observers in both groups; for the Attention group the data were close to the unity line (right column); for the Neutral group they were more disperse. Note that all Learners also had faster RT, whereas Non-Learners did not. Thus, we can rule out speedaccuracy tradeoffs for all observers.

To evaluate VPL transfer, observers must first show learning at the trained conditions. To assess whether training with exogenous attention promotes generalization to untrained spatial locations, we conducted a 3-way (Session: pre-test, post-test X Diagonal: trained, untrained X Group: Neutral, Attention) mixed ANOVA of d' for the Learners in each group (Figure 3, top row). Critically, there was a significant 3-way interaction among Session, Diagonal and Group $\left[F(1,12)=5.94, p=.031, \eta^{2} G=.037\right]$ : At the trained diagonal, both groups showed higher performance at post-test than pre-test [Neutral group: $M=1.83 \pm .13$ vs. $M=1.10 \pm .18, t(6)=3.60, p=.011,95 \% \mathrm{Cl}$ [.24,1.23]; Attention group: $M=2.12 \pm .19$ vs. $M=1.45 \pm .17, t(6)=3.29, p=.017,95 \% \mathrm{Cl}[.17,1.18]]$. But at the untrained diagonal, only the Attention group showed significantly higher performance at post-test than pre-test [ $M=2.09 \pm .23$ vs. $M=1.20 \pm .13 ; t(6)=4.13, p=.006,95 \% \mathrm{Cl}[.36,1.42]]$; the Neutral group showed similar performance in both sessions $[M=1.41 \pm .19$ vs. $M=1.16 \pm .15$; $t(6)=1.12, p=.31,95 \% \mathrm{Cl}[-.30, .80]]$. 2-way (Session X Diagonal) ANOVAs within each group 
revealed that whereas the Neutral group showed a significant Session $x$ Diagonal interaction $\left[F(1,6)=14.1, p=.009, \eta^{2} G=.090\right]$, the Attention group only exhibited a main effect of Session $\left[F(1,6)=21.6, p=.004, \eta^{2} G=.39\right.$; both main effect of Diagonal and interaction $\left.F<1\right]$. Consistent with these findings, the complementary 2-way (Diagonal $X$ Group) mixed ANOVA of normalized proportion change revealed that only the 2-way interaction between Diagonal (trained, untrained) and Group (Neutral, Attention) was significant $\left[F(1,12)=9.46, p=.010, \eta^{2} G=.13\right]$.

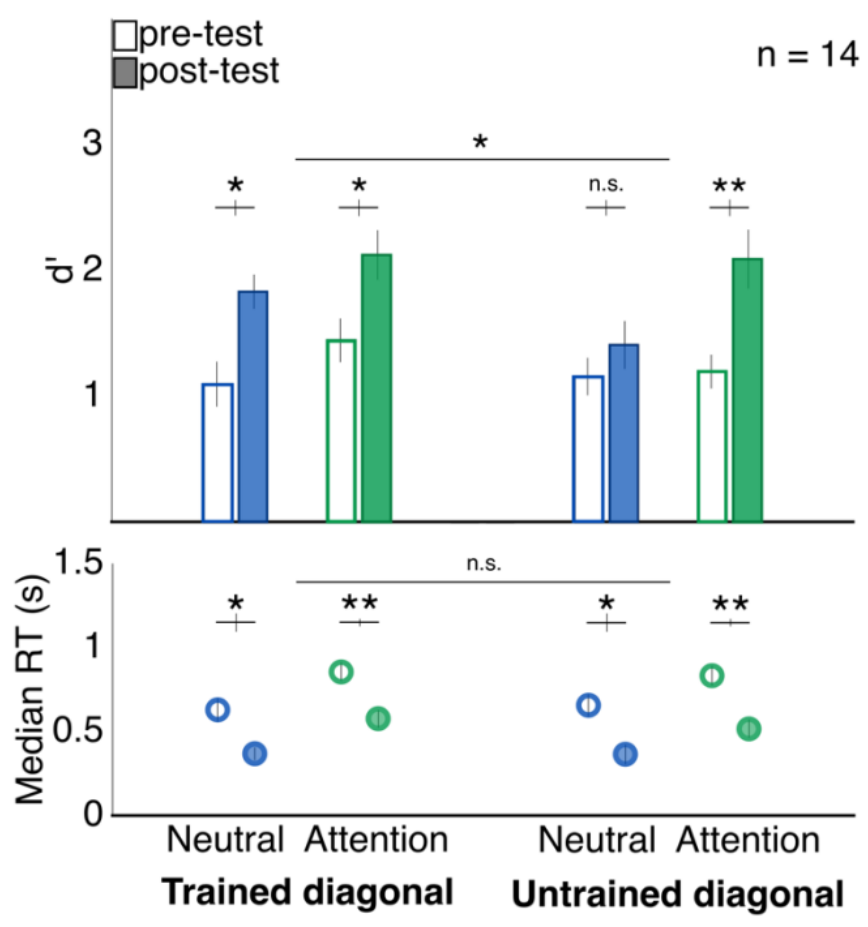

Figure 3. Pre-test and post-test performance for the Learners, collapsed across eyes and split by diagonal and group. Error bars represent \pm 1 within-subject standard error [53]. ${ }^{*} p<.05,{ }^{* *} p<.01$.

Corresponding analyses of the secondary variable of median RT only revealed a significant main effect of Session $\left[F(1,12)=39.1, p<.001, \eta^{2} G=.16\right.$; all other main effects and interactions: $\left.p>.3\right]$. (Figure 3, bottom row). The 2-way (Diagonal X Group) mixed ANOVA of normalized proportion change in $\mathrm{RT}$ revealed no significant main effects or interaction [all $F<1$ ].

logMar visual acuity. We also investigated whether training on a contrast sensitivity task in the periphery would translate to amblyopic and/or fellow eye improvements in logMar acuity at the fovea, the visual dimension traditionally used to diagnose amblyopia. A 3-way (Session: pre-test, post-test X Eye: amblyopic, fellow X Group: Neutral, Attention) mixed ANOVA of observers' logMar visual acuity (Figure 4 for individual scatter plots; higher values are worse) revealed highly significant main effects of Session $\left[F(1,17)=21.5, p<.001, \eta^{2} G=.10\right.$; pre-test $M=.271 \pm .05$, post-test $M=.147 \pm .04]$, Eye $\left[F(1,17)=42.2, p<.001, \eta^{2} G=.57\right.$; amblyopic eye $M=.425 \pm .04$, fellow eye $M=-$ $.007 \pm .02]$, and a marginal 2-way interaction of Session and Eye $\left[F(1,17)=4.25, p=.055, \eta^{2} G=.03\right]$, indicating that training on a contrast sensitivity task also improved acuity. Because some condition values were negative, we added a fixed constant (0.25) to all condition values of each participant before computing normalized proportion change so that all resulting values were positive; see the original values in the left and middle columns of Figure 4. The pattern of results was the same for different constant values, indicating that our results did not depend on the specific constant value we selected. None of the main effects or interactions in the 2-way [Eye X Group) mixed ANOVA of 
normalized proportion change was significant [all $p>$.2], indicating that there was similar transfer of improved acuity to the untrained fellow eye in both groups. The pattern of results was the same when including only the Learners.
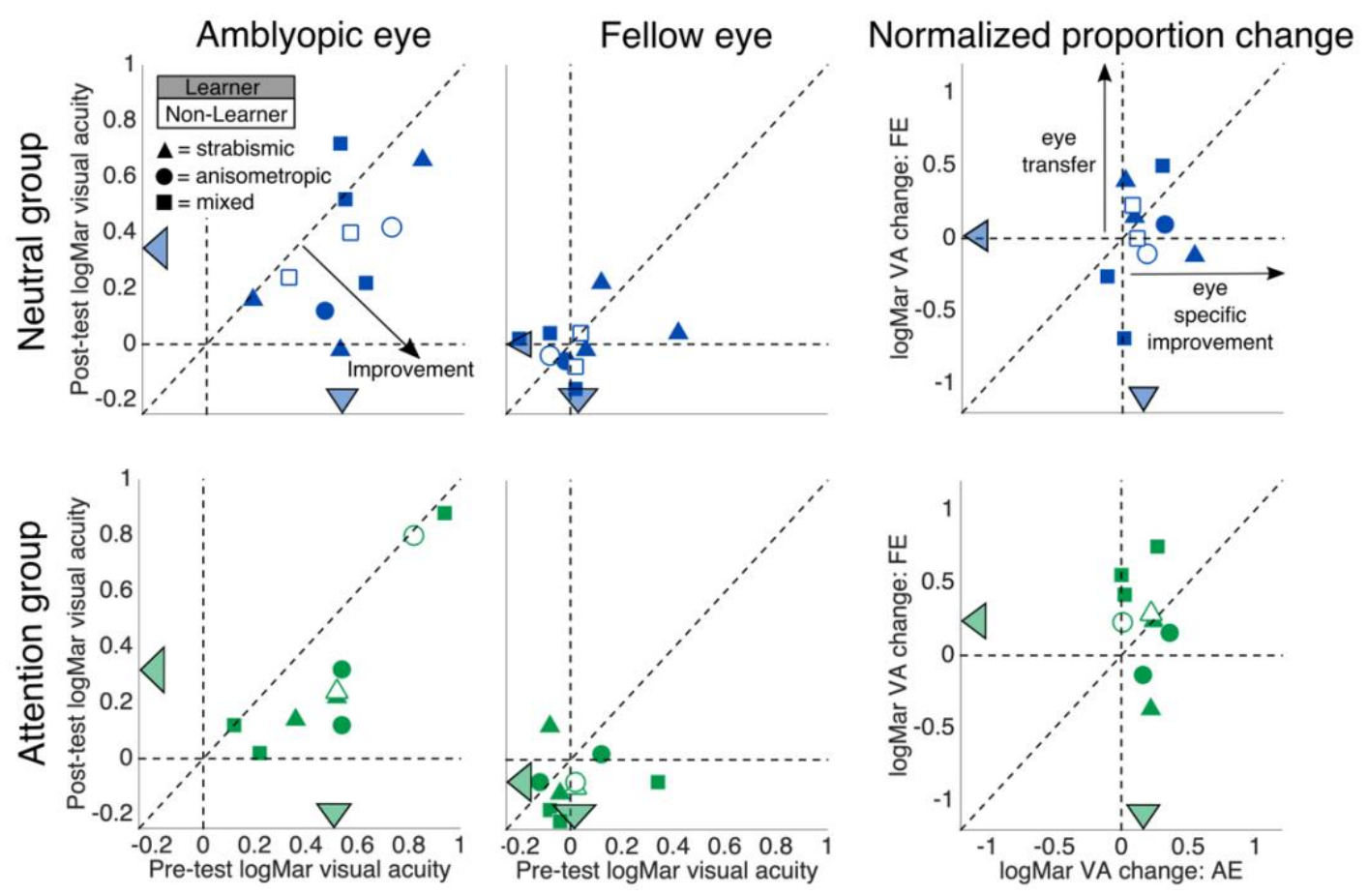

Figure 4. logMar visual acuity for 10 observers in the Neutral (top row) and 9 observers in the Attention group (bottom row) at post-test versus pre-test for the amblyopic eye (left column), fellow eye (middle column), and the relative normalized proportion change in each (right column). The colored arrows pointing to the axes indicate group means, and their widths represent \pm 1 within-groups SEM for each condition [53].

We also assessed whether and to what extent training reduced the interocular difference in visual acuity (i.e., the amblyopic "depth"), a common metric for quantifying amblyopia severity. We calculated the proportion of amblyopic depth corrected in each observer as the difference in amblyopic depth at post-test compared to pre-test, divided by the amblyopic depth at pre-test. One sample t-tests revealed that visual training corrected for a significant proportion of amblyopic depth and ameliorated amblyopic severity in both groups [Neutral: $M=.508 \pm .19 ; t(9)=2.70, p=.024,95 \%$ $\mathrm{Cl}[.08, .93]$; Attention: $M=.519 \pm .21 ; t(8)=2.48, p=.038,95 \% \mathrm{Cl}[.04,1.0]]$.

VPL transfer to other untrained tasks. Several VPL studies with amblyopic adults have reported generalized improvements beyond the trained task for some untrained features (e.g., spatial frequencies and orientations) and visual dimensions (reviews [9,12,20-23]). Thus, we also assessed whether, after training on a contrast sensitivity-dependent orientation discrimination task in the periphery, observers would show improvement in a curated battery of visual tests assessing broadband foveal contrast sensitivity (Figures S2-S5), crowding (Figure S6) and stereoacuity (Figure S7).

Figure 5 provides a summary of the results of a series of 3-way (Eye: amblyopic, fellow X Group: Neutral, Attention X Session: pre-test, post-test) mixed ANOVAs for all untrained tasks, which were carefully chosen to assess some of the classic deficits in amblyopia. In all tasks, the amblyopic eye performed worse than the fellow eye (i.e., significant main effect of Eye), verifying that observers were amblyopic and that there was a significant disparity between their eyes on a variety of visual 
dimensions. The main effect of Group was never significant, showing that both groups were composed of individuals with similarly severe amblyopia, and thus had equal opportunity to improve with training. Observers improved at post-test for all tasks except for the one that measured the critical spacing of crowding. There was a significant 3-way interaction among Eye, Group and Session for the AULCSF, a measure of foveal broadband contrast sensitivity, and a significant 2-way interaction of Eye and Group for the complementary measure of normalized proportion change in the AULCSF (Figure 5b); the Neutral group only showed modest improvement in their amblyopic eye, whereas the Attention group showed similar but modest improvements in both their amblyopic and fellow eyes. The amblyopic eye improved more than the fellow eye for the Pelli-Robson contrast sensitivity task, a coarser measure of foveal broadband contrast sensitivity, as shown by a significant 2-way interaction of Session x Eye and main effect of Eye for normalized proportion change in Pelli-Robson values (Figure 5b). For the detailed methods and results for all of these untrained tasks, see the Supplemental Information.

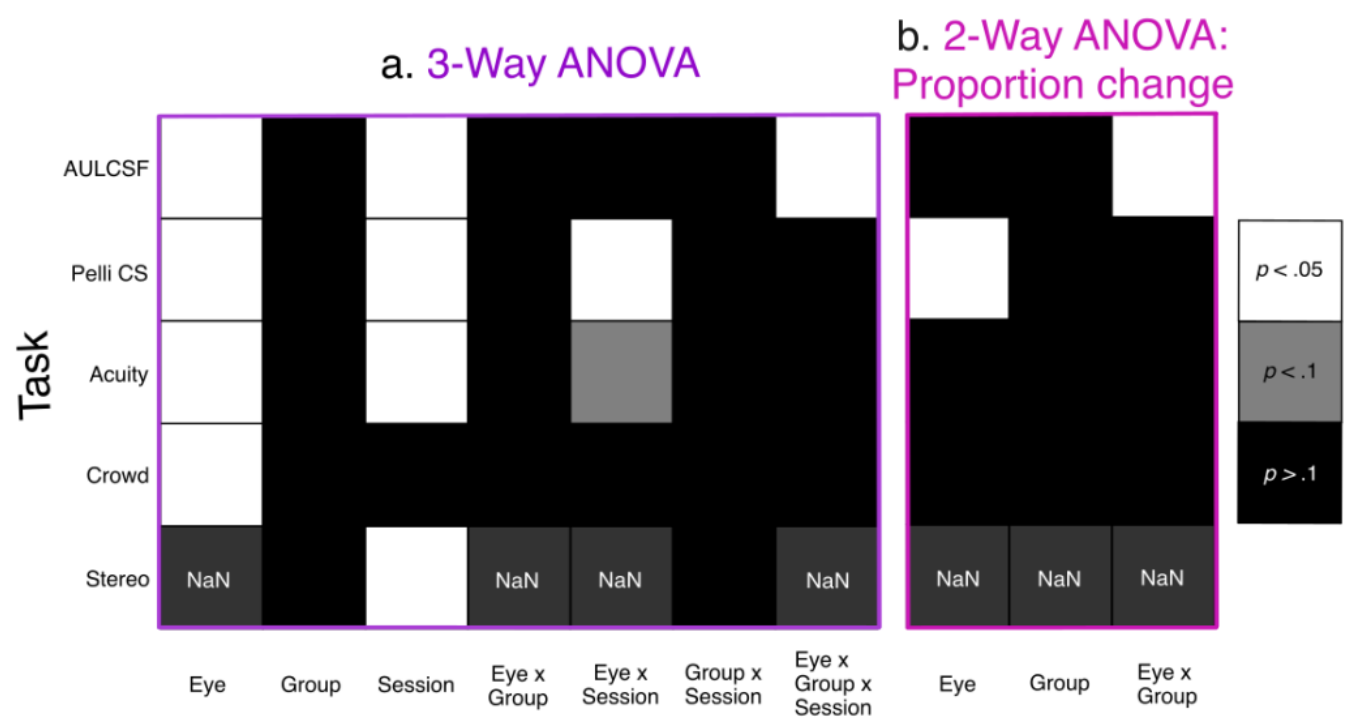

Figure 5a. A graphical representation of the results of a series of 3-Way ANOVAs for all untrained visual tasks (left table) and b. 2-WAY ANOVAs for their corresponding normalized proportion change values (right table). The shading of each square represents the statistical significance of the main effect or interaction in each of the respective tasks. See also Figures S3-S7.

\footnotetext{
Evidence of generalized learning within the same observers: Positive correlations in magnitude of proportion improvement across certain tasks. To assess whether and how performance changes in one task predict changes in another within the same observer, we calculated a Pearson's correlation matrix. Figure S8 is a graphical representation of the significance of correlations of normalized proportion change across all tasks for the amblyopic eye (bottom left triangle) and fellow eye (upper right triangle), combining across both groups. The majority of significant correlations showed a positive relation, i.e., improvement in one was correlated with improvement in another. Improvements in the amblyopic eye were significantly correlated across several tasks, with fewer significant correlations for the fellow eye. We detected more significant correlations between changes in the amblyopic than fellow eye. We do not interpret this to mean that proportional changes were not correlated across tasks for the fellow eye. Rather, we think the results are at least partially due to a floor/ceiling effect; as the fellow eye was already high performing and had a much smaller range for improvement, the normalized proportion change values would be artificially compressed, subsequently diminishing our likelihood of detecting a significant correlation.
} 
Notably, improvement at the trained diagonal on the main task was positively correlated with improvement at the untrained diagonal in both eyes. Improvement in the amblyopic eye at the trained diagonal was also marginally correlated with visual acuity improvements and significantly correlated with stereoacuity improvements. Furthermore, more improvement at the untrained diagonal with the amblyopic eye predicted greater improvements in visual acuity, crowding and stereoacuity. Improvements in amblyopic eye acuity were significantly correlated with crowding improvements and marginally correlated with stereoacuity improvements. Finally, improvements in crowding were significantly correlated with improvements in stereoacuity. Note, however, that at the group level, mean changes in the critical spacing of crowding did not reach statistical significance.

Although some correlations were found between tasks that were more intuitively associated as they indexed the same dimension of vision, i.e., significant correlations between improvement in the main task at the trained and untrained diagonal, that was not always the case. For example, improvement at the untrained diagonal seemed to be the most common predictor of improvements in other visual dimensions, i.e., acuity, crowding, and stereoacuity. Thus, in general the same observers showed a similar degree of improvement across tasks, evidence for widespread improvement not specific to certain locations, tasks or visual dimensions. Despite the relative variability in how much observers improved overall, there was significant within-subjects consistency across tasks.

Pre-test performance correlates with magnitude of improvement. In an exploratory analysis, we assessed whether pre-test performance in any of the tasks correlated with normalized proportion change in the same task or any others. The results of all possible Pearson's correlations for the amblyopic and fellow eyes of all observers collapsed across group are shown in Figure S9. Note that the majority of squares show a negative relation, indicating that observers who were worse to start improved more, in agreement with previous studies (reviews[20,24]). For example, lower AULCSF values at pre-test predict greater proportional improvement in the AULCSF in both eyes, as well as greater improvements in visual acuity and crowding in the amblyopic eye. The result is the same for Pelli-Robson contrast sensitivity; a lower Pelli-Robson score at pre-test is associated with larger contrast sensitivity gains in both eyes. Further, in the fellow eye, worse visual acuity and larger critical spacing values correlate with greater improvements in visual acuity and crowding, respectively.

\section{DISCUSSION}

Two groups of adults with a similar severity of amblyopia trained during approximately ten 1-hour sessions on an orientation discrimination task. Their performance was well equated before training by individually adjusting the stimulus contrast presented to each eye for each observer. The pretest and post-test were identical for both groups, and during the training days the only difference was that one group trained with valid peripheral exogenous precues (Attention group), whereas the other trained with uninformative neutral precues (Neutral group).

Our training protocol successfully induced VPL. After training the amblyopic eye, both eyes of most observers in both groups exhibited similar performance improvements -higher d' and faster RT- at the trained diagonal; training with attention did not potentiate the overall improvement at the trained diagonal, consistent with [28-30]. Our study contributes to a rich VPL literature in both neurotypical humans (reviews[1-5]) and those with amblyopia (reviews[10,12,20-23]) demonstrating impressive neuroplasticity in the adult brain. 
There is evidence that the temporal dynamics of visuospatial processing may be altered in amblyopia (e.g.[35,54]). Our primary goal was to assess whether and how spatial attention precues modulate discrimination performance changes during visual training with amblyopic adults, in spite of their known early sensory processing deficits. Therefore, we designed a task to promote changes in d' rather than RT: Observers were explicitly instructed to be as accurate rather than as fast as possible, and were forced to wait before responding to ensure all observers had adequate time to accrue asymptotic levels of sensory information. Most critically, there were no speedaccuracy tradeoffs -observers were fastest during conditions in which they were most accurate.

There was complete interocular transfer of VPL; both eyes exhibited similar magnitude VPL in both groups of observers. Despite being relatively stronger than the amblyopic eye, the dominant, untrained fellow eyes of adults with amblyopia possess significant room for improvement; they underperform in a variety of visual tasks relative to typically developed eyes [19], which exhibit VPL themselves (reviews[1-5]). Some monocular training studies have found eye-specific VPL (review[1]), but others in neurotypical adults [55] and patients with visual disorders [56] have found interocular transfer. Overall, our study strengthens the claim that VPL is not specific to the trained eye in amblyopia and leads to significant improvements in the untrained fellow eye (reviews[20,21,23]).

Exogenous attention helps overcome location specificity in adults with amblyopia. Importantly, this is the first study to investigate the role of exogenous attention during VPL in a special population of adults with atypically developed, but highly plastic, visual cortex. Despite similar improvements at the trained diagonal in both groups, the Learners of the Neutral group exhibited location specificity -no significant learning at the untrained diagonal in either eye. In contrast, the Attention group showed VPL location transfer -similar d' improvement in both eyes at the trained and untrained diagonals. We found that spatial attention helps overcome location specificity, consistent with the few studies that have isolated the role of spatial attention during VPL and location transfer in neurotypical adults [28-30].

Both groups were only presented with neutral cues during the pre-test and post-test sessions. The only difference was the attention precue during training -either neutral or peripheral, transiently shifting their attention to the target location. Given that the training locations were known in advance, observers could have allocated their endogenous attention to both potential target locations to improve performance. Any such benefits would have been similar across training in both groups. Moreover, the 134-ms delay between the peripheral precue and stimuli presentation was too short for endogenous attention to be selectively deployed to the target location (which takes $\sim 300 \mathrm{~ms}$; review[25]). Thus, the fact that both groups show similar performance at the trained diagonal but differential improvement at the untrained can only be attributed to differences in exogenous attentional allocation during training.

Even though, as we explained in the Introduction, it was unclear whether exogenous attention could have any effect on VPL in the amblyopic brain, amblyopic observers who trained with exogenous attention demonstrated even greater VPL; amblyopes in the Neutral group only improved in the main task at the trained diagonal, but those trained with attention improved at both diagonals. These findings are encouraging, as they demonstrate that neuroplasticity in the adult amblyopic brain may extend further than previously known.

Promoting transfer over specificity. A goal of visual training in amblyopia and other visual disorders is to maximize improvement in every way - across spatial locations, features, tasks, visual dimensions and eyes. Thus, our design included several aspects known to promote greater transfer of learning over specificity: 
(i) simply having exposed stimuli at the untrained diagonal in the main task during the practice, adaptive staircase and pre-test blocks may have facilitated transfer [e.g.,57].

(ii) training at two locations along one diagonal, rather than at one location, likely promoted generalization [e.g.,28-30].

(iii) training on stimuli of differing difficulty (a higher and lower contrast) that were randomly intermixed within each block. Including some easier trials promotes location and orientation transfer (e.g.[58]; review[59]). Likewise, blocks consisting of several short staircases, which contain a higher proportion of easier trials, have shown to facilitate VPL transfer in neurotypical adults (e.g.[60]).

(iv) training on contrast-based tasks leads to greater degrees of improvement and transfer than training on acuity-based tasks in adults with amblyopia (reviews[20,23]).

There are many other known factors to influence VPL transfer, including sensory adaptation [61], task precision [62], length of training [63], and sensory uncertainty of stimulus features in visual search [64]. As all of those factors were kept constant across our two groups, they cannot explain different patterns of VPL transfer.

Individual variability in VPL. As often the case with perceptual learning studies (reviews[3-5,15]) and particularly with amblyopia (reviews[9,12,20-23]), there was a substantial degree of individual variability. Three observers in both the Neutral and Attention groups (i.e., the "Non-Learners") did not improve in d' with training at the main task even at the trained diagonal. Correspondingly, those same observers did not show improvement at the untrained diagonal; an observer cannot show transfer of learning if they did not learn in the first place. Even among the Learners, there was variability in the degree of transfer, whether none, partial or complete (i.e., equal learning at the trained and untrained diagonals), to the untrained diagonal across observers in both groups. Critically, the percentage of Learners who exhibited partial or complete transfer of VPL was significantly greater in the Attention group (6/6, 100\%) than the Neutral group $(3 / 7,43 \%)$. This finding is similar to other studies that have reported notable intra-individual variability in both the magnitude of overall VPL and degree of transfer, but find that reliable patterns emerge at the group level (e.g.[28], reviews[12,22,23]).

Optimal amount of training. There is evidence that the number of training hours that it takes for an observer to achieve asymptotic performance directly scales with the severity of their deficit [65]. Thus, an observer with mild amblyopia may asymptote within ten training sessions, whereas a more severe observer may barely start performing above chance within the same number of sessions and practice blocks. Thus, a standard number of training sessions would not likely capture the full learning curves for each individual observer. This mismatch between an individual's optimal number of training sessions and our standard of ten training sessions may partially explain why most observers exhibited significant VPL while a minority did not.

Training protocol. Our visual training task was relatively demanding; observers were required to discriminate a peripheral target while maintaining fixation within a relatively tight fixation window. If their eye moved outside of the window, the trial would be cancelled, and they would have to redo the trial at the end of the block. As some observers suffered from greater fixational instability, especially those with the strabismic ("lazy eye") or mixed subtypes of amblyopia, not all could complete the same number of training blocks each day before becoming fatigued. In these few cases (5 out of 20), we had to spread the 80 training blocks across more than ten sessions. Importantly, the average number of training sessions per group did not significantly differ from each other.

Another source of variability was the different number of days that passed between training sessions. We strongly recommended that no more than two days pass between training sessions, as sleep consolidation and consistency have been shown to be critical factors in PL [66], but 
occasionally there were unavoidable scheduling conflicts. Fortunately, the average number of days that both groups of observers took to complete training did not significantly differ from each other (Neutral group: $M=10.8 \pm .490$ sessions, Attention group: $M=10.1 \pm .100$ sessions; $t(18)=1.40$, $p=.179$, spread across $21.3 \pm 1.10$ days and $18.0 \pm 1.56$ days, respectively; $t(18)=1.30, p=.209$ ). Other human factors out of the experimenters' control, such as observers' quality of sleep (common sources of variability in all VPL studies), may have contributed to the individual differences in VPL.

Modulatory role of amblyopic severity. Previous studies have found initial severity to be the biggest predictor of overall improvement (e.g.[67]; reviews[20,21,23]). Importantly, there were no pronounced differences in the composition of amblyopic severity (amblyopic eye visual acuity), and depth (interocular acuity difference) between the two groups (see Table 1). Across all visual tasks and observers, the amblyopic eye was worse than the fellow eye, providing confirmatory evidence for observers' amblyopia diagnosis, and that the amblyopic contrast sensitivity deficit extends into the parafovea. As expected, all observers required more contrast in their amblyopic eye than fellow eye to complete the task with similar performance; but most importantly, the required stimulus contrast for each eye did not significantly differ between groups. Interestingly, within each group we found convergent evidence that those observers with worse performance generally improved more in proportion to their initial performance across various tasks in both eyes (see Figure S9), probably because they had more room for improvement.

Amblyopia subtypes. It has been proposed that the various subtypes of amblyopia (i.e., anisometropic (refractive), strabismic ("lazy eye"), deprivational, or mixed) actually represent different visual populations, whose unique etiologies result in distinct constellations of visual deficits $[10,12,36,68,69]$. For example, strabismic amblyopes are thought to have better contrast sensitivity but worse visual acuity than anisometropic amblyopes. Our sample size did not include enough of each type to perform formal statistical analyses (see all scatter plots with different symbols for subtypes), but through visual inspection we did not observe any clear patterns in the magnitude and transfer of VPL for the main task or all untrained tasks according to subtype. Although it was not the main question for this study, understanding potential differences in VPL effects between the amblyopia subtypes remains an interesting empirical question for future research.

Differences in number of fixation breaks. Given their known issues with fixation stability, particularly for strabismic and mixed observers, one may wonder whether fixation breaks during the main task significantly affected observers' performance [12,54], as a higher preponderance would have extended the length of each training block, which was likely frustrating and taxing. We counted the number of fixation breaks within each block, and conducted complementary analyses to see how the total may have evolved across training and during the pre-test and post-test. We did not observe a clear relation between the total number of fixation breaks during a block and overall performance within the same observers. Further, the total number of fixation breaks did not systematically change across training, nor did it significantly differ between the pre-test and posttest. Regardless, it is unclear that a less stable gaze would have significantly affected their performance, as the contrast sensitivity of amblyopes does not improve even when retinal image motion is eliminated by image stabilization [70]. The fact that we did not observe significant changes in all tasks, i.e., no reductions in the critical spacing of crowding, also supports the idea that task improvements were due to underlying changes in visual sensitivity, and not attributed to alternative explanations such as improved gaze stability or accommodation. Together, the results indicate that individual differences in the ability to consistently fixate on the central cross cannot explain the observed variability in VPL across conditions and tasks. 
Concomitant gains in visual acuity. Given that studies in which observers trained on a contrast sensitivity-dependent task have often shown the largest magnitude task transfer of VPL to visual acuity (review[23]), we had a particular interest in measuring the interocular difference in visual acuity at the fovea, a classic diagnostic marker of amblyopia. VPL studies have shown dramatic changes in amblyopic eye acuity; e.g., an overall mean improvement of .17 logMar, with a third of participants achieving gains >.2 [21]. Most of our observers showed concurrent improvements in visual acuity: $\sim 2$ lines in their amblyopic eye in both groups, with smaller improvements for the fellow eye ( 0.5 line for the Neutral group and $\sim 1$ line for the Attention group), which had less room for improvement. Remarkably, training reduced the interocular visual acuity difference, or amblyopic depth, by $\sim 50 \%$ across both groups. This study contributes to a growing literature (reviews[12,20,22,23]) indicating a close link between improvements in contrast sensitivity and acuity in the brain.

Notably, all of our so-called "Non-Learners" in both groups, i.e., those who did not show significant improvement in the main contrast sensitivity task at the trained diagonal, did show some improvement in amblyopic eye visual acuity (Figure 4). Acuity is a clinically defining deficit of amblyopia and the dimension which most often seems to show concomitant improvements regardless of the training task (reviews[12,20,22,23]). Whereas we do not have an explanation for this pattern, our findings suggest that the time-course or mechanisms of VPL for contrast sensitivity and acuity may differ in the amblyopic brain and lead to faster improvements in acuity.

Selective attention in amblyopia. Some researchers have attributed differences in performance between amblyopic and neurotypical adults on a few higher-level psychophysical tasks -i.e., the attentional blink, numerosity estimation, and multiple object tracking-to an attention deficit in amblyopia. Critically, most have only inferred the role of attention in each of their tasks without directly manipulating it (see [16,17] for more details). More recently, it has been hypothesized that increased fixation instability (particularly for strabismics) leads to higher rates of microsaccades, or fixational eye movements $<1^{\circ}$, which results in an amblyopic deficit in selective attention [54]. However, there is controversy as to whether amblyopes reliably generate higher rates of microsaccades (see Discussion of [18]). Further, there is not a direct or causal correspondence between eye movements at any scale and voluntary or involuntary attention (review[71]); neurotypical (review[25]) and amblyopic (e.g.[16,18]) observers can covertly attend elsewhere from where they are fixating, and neurotypical observers can selectively attend within the high-acuity foveola without fixational eye movements and even with retinal stabilization [72].

There is evidence that the neural mechanisms and correlates of voluntary attention differ in the amblyopic brain $[17,34,35]$. A study measuring the full contrast-response functions of amblyopic monkeys for a motion direction discrimination task found that, unlike the fellow eyes which exhibited only contrast gain, the amblyopic eyes showed both contrast and response gain, perhaps in a compensatory fashion [17]. A different study with strabismic adults measured steady-state visual evoked potentials while observers were cued to voluntarily attend one of two hemifields to detect a contrast increment in a peripheral grating [15]. They found reduced attentional modulation of neural activity both in striate and extrastriate cortex corresponding to the amblyopic eye, but only in the extrastriate cortex corresponding to the fellow eye. More recently, a study measuring eventrelated potentials found some delayed latencies and reduced peak amplitudes in components corresponding to the amblyopic eye, which have been associated with various stages of the visual processing hierarchy [35]. The authors reported a higher d' for the cued hemifield compared to the uncued. Unfortunately, the psychophysical methodology of both EEG studies severely limits our ability to interpret specifically what and how atypical neural signaling may correlate with deficits in amblyopic observers' attentional rather than visuospatial processing per se. Both employed detection tasks but did not report criteria, thus rendering it impossible to distinguish whether changes in d' were due to shifts in perceptual sensitivity to the targets or to observer criterion (i.e., 
likelihood of reporting “yes"). Moreover, neither reported performance during an uninformative neutral or misleading invalid cueing condition. Without a comparison condition, we cannot behaviorally verify the extent to which observers deployed their voluntary attention according to the valid cue instruction.

Only a few studies have employed precues to directly manipulate covert attention in amblyopia [16$18,32,34,35]$. Altogether, they show that amblyopic adults [16,32], children [18] and monkeys [17] benefit when spatially cued to voluntarily [16-18,32] and involuntarily [16] attend to a task relevant spatial location and show a behavioral cost when their attention is shifted elsewhere [16,32]. It is clear that covert selective spatial attention is behaviorally functionally intact in amblyopia, even if through alternative and potentially compensatory neural mechanisms. Given that we found differences in VPL transfer at post-test between our two groups, whose training only differed in the location of the exogenous precue (adjacent to the target or fixation cross), the current study provides further evidence that the adult amblyopic brain can effectively use a spatial cue during perceptual training to induce meaningful changes in perceptual processing that persist even when the cue is no longer onscreen.

It is important to note that in our current study we employed peripheral exogenous precues during an orientation discrimination task. All but one of the studies isolating the role of attention in amblyopia have explicitly manipulated voluntary attention. The only study with exogenous attention found that cues adjacent to the target location benefited orientation discrimination performance whereas those far away disadvantaged performance. These effects were as pronounced when observers used their amblyopic eye as when they used their fellow eye, and also as pronounced as the corresponding effects of neurotypical age- and gender-matched controls [16]. The effectiveness of transient cues presented exclusively to the weaker eye during push-pull binocular training programs for re-calibrating interocular balance [10] also suggests that the amblyopic eye robustly responds to bottom-up or stimulus-driven (exogenous attention) cues. More research should be done to fully characterize the behavioral effects and largely unknown neural correlates of exogenous and endogenous attention in amblyopia using different visual tasks (e.g., visual search, the attentional blink paradigm, motion object tracking), and under conditions of increasing task difficulty and/or greater attentional load.

How attention may generalize learning to untrained locations. From a mechanistic standpoint, modeling studies have revealed substantial signal attenuation, increased additive internal noise, and weaker perceptual templates in the amblyopic brain (reviews[12,36]). Thus, as in other amblyopia VPL studies (e.g.[65]), visual training may have acted to reduce this elevated internal noise and retune observer's perceptual templates.

There are several conceptual (e.g.[5]) and computational models of VPL (review[3]). Researchers have speculated that attention plays a critical role, but most have discussed it in terms of task relevance or in selectively "gating" what gets learned [3,5], and none has explicitly modeled the role of spatial attention on VPL. It is notable that no current theory or model of visual perceptual learning can explain why or how exogenous attention generalizes VPL to untrained spatial locations in the amblyopic brain. Regardless, below we highlight some models and how they may relate to our findings:

A rule-based learning model suggests that VPL primarily involves learning rules for performing the task efficiently, and that specificity is a consequence of an inability to link signals from early visual cortex that represent untrained stimuli to the learned rule scheme (e.g.[73]). This model cannot account for our findings, as the model predicts that: (a) for transfer to occur, the rule scheme must be learned before exposure to untrained locations or features. But for both groups, we exposed the 
untrained diagonal only during the pre-test, not during training; (b) transfer is prevented at unstimulated locations because they are suppressed. Accordingly, the Attention group should show more specificity, not transfer as we observed, because exogenous spatial attention decreases neural activity at unattended locations (e.g.[41,45,51]).

The Reverse hierarchy theory postulates that VPL is a top-down guided process that begins with higher-level regions and progresses to lower-level sensory regions with more stimulus repetitions and/or challenging perceptual tasks; location-specific learning is associated with plasticity in early visual regions and is more likely to occur for difficult tasks with noisy sensory signals [74]. According to this framework, we speculate that location transfer in the Attention group could have resulted from exogenous attention improving the target's signal-to-noise ratio and increasing its visibility, thereby making the task easier during training. Attention-induced modification would thus be more likely to occur in higher-level decision-making areas, e.g., lateral intraparietal cortex (LIP), which contain broadly tuned, bilateral receptive fields, therefore enabling learning effects to transfer to untrained locations as far as in the opposite hemifield $[1,3]$.

The Integrated Reweighting Theory proposes to account for specificity and transfer in VPL (review[3]). To explain transfer across retinotopic locations, this model incorporates higher-level, location-independent representations with lower-level, location-specific representations that are both dynamically modified in VPL. According to this theory, specificity of VPL to the trained diagonal in the Neutral group would likely be due to the reweighting, or enhanced readout, of the lower-level, location-specific representations. Transfer in the Attention group would likely be due to reweighting of higher-level, location-invariant representations of the decision stage [3]. Currently, no VPL reweighting model explicitly models the role of selective attention. Future computational modeling studies may illuminate the specific mechanism(s) through which attention may promote greater change at higher levels of the model hierarchy.

Where are the neural correlates of VPL in amblyopia? VPL that is specific to locations and features is often considered to take place in primary visual cortex (reviews[1,4]), as V1 neurons respond to precise retinal locations and simple visual features. The finding that our observers demonstrated complete interocular transfer of VPL suggests that the neuronal correlates of this learning most likely reside in cortex, where the input from the two eyes first combines. Binocular convergence first occurs in early visual cortex. But given that there are fewer binocularly driven neurons (reviews[11,12]) and abnormal patterns of binocular interactions in amblyopic V1 (reviews[10,33]), VPL could have occurred either at primary visual cortex or downstream in higherorder vision or decision-making areas. Our findings suggest that attending to the target location during training may have increased neural activity in occipital cortex (e.g.[39,41,44,45,51]) and altered the distribution of VPL changes to be biased towards higher brain areas whose responses are less tuned to specific spatial locations, features and/or eyes. It is now considered that VPL for any task is widespread, likely involving several areas, and that the pattern of VPL changes and their neural correlates will differ according to the unique training protocol, as well as type and difficulty of the task employed in each study (e.g.[75]; reviews[2-5]).

Translational implications for rehabilitation of visual disorders. With early detection and compliance, there are several treatments for children with amblyopia (e.g., refractive correction, surgical realignment of the eyes, patching) that largely mitigate the most severe symptoms from persisting into adulthood (reviews $[9,12,20]$ ). In contrast, until about a decade ago, treatment options for adults were largely thought to be futile. However, VPL studies have advanced our understanding of the impressive remaining neuroplasticity in the (a)typically developed adult brain and identified visual training as a promising rehabilitative tool. Perceptual training improves visual performance in individuals with amblyopia (reviews[9,10,12,20-23]), visual acuity in presbyopia [76], visual acuity and contrast sensitivity in individuals with optical aberrations [56], and visual 
motion discrimination in people with $\mathrm{V} 1$ damage $[77,78]$. But the prognoses for most visual disorders remain poor and achieving any improvements in daily vision can take a long time.

Most current VPL protocols that promote location transfer in neurotypical adults (e.g.[6-8]) are limited in their practicality for clinical populations; they would demand a lot of effort from an already bioenergetically taxed system, and patients would have to practice more than one task to achieve maximal transfer. Here we present a simple and elegant possibility for improving visual training efficiency -training with exogenous attention [27,28,30,31], which does not require any special instruction, or even awareness of the peripheral cue. Observers do not have to train on any extra trials, tasks, or with more cognitive effort. Attention helps overcome specificity by promoting transfer of learning beyond trained spatial locations.

Conclusions. This study reveals that exogenous attention generalizes the effects of perceptual training to untrained spatial locations in adults with amblyopia and potentially could do so for other groups with visual disorders.

Limitations of the study. Due to the COVID lockdown, we were unable to collect the post-test data for one observer in the Attention group for all tasks in the visual battery. We were also unable to bring observers back for a 6-month follow-up to see how much of the learning was retained after an extended period, as we had originally planned.

\section{MATERIALS \& METHODS}

Observers. Twenty adults with amblyopia and no other visual disorder or disease participated in the study (see Table 1 for demographic and clinical information). Before training, all observers exhibited $\geq$ two-line interocular difference in best-corrected EDTRS logMar acuity, in which each line equals $0.1,0$ is equivalent to 20/20 Snellen acuity, and a higher score is worse [Neutral group $M=.546 \pm .062$, Attention group $M=.594 \pm .088 ; t(18)=-.462, p=.650]$. All experimental procedures were in agreement with the Helsinki declaration and approved by the New York University Institutional Review Board. All observers were naïve to the experimental hypotheses and signed a written consent.

Apparatus \& setup. All computer-based tasks shared the same setup. Experiments were run from a 21.5" Apple iMac 3.06 GHz Intel Core 2 Duo Desktop using MATLAB (MathWorks, USA) in conjunction with the MGL toolbox (http://justingardner.net/mgl). They were presented at a viewing distance of $114 \mathrm{~cm}$ on a $40 \times 30 \mathrm{~cm}$ IBM-P260 CRT monitor (1280x960 pix resolution, 90-Hz refresh rate), calibrated and linearized using a Photo Research PR-650 SpectraScan Colorimeter.

Eye movements were monocularly monitored using an EyeLink 1000 Desktop Mount eye tracker (SR Research, Canada) with a $1000-\mathrm{Hz}$ sampling rate. We used a 5-point display to calibrate the eyetracker at the beginning of every block and within blocks as needed to minimize fixation breaks. Observers' logMar visual acuity was measured using the $2 \mathrm{M}$ Original Series EDTRS Chart R (Precision Optics Ltd., CO)[79].

Procedure. There were three phases to the study, which took place across at least 17 different sessions (Figure 1b). During the pre-training phase (Phase I; days 1-4), we prescreened observers for qualification in the study and measured their abilities on a variety of visual tasks. During Phase II, observers performed the same training task for $\sim 10$ sessions while being presented with either a neutral (Neutral group) or valid (Attention group) attention precue. The three final sessions of the post-training phase (Phase III; days 15-17) were identical to those of 
Phase 1, except these sessions did not include the adaptive staircase session for measuring contrast thresholds from day 2 and were administered in reverse order.

\begin{tabular}{|c|c|c|c|c|c|c|c|c|c|}
\hline Participant & Gender & Age & $\begin{array}{l}\text { Amblyopic } \\
\text { eye (LR) }\end{array}$ & Depth' 1 & Severity ${ }^{2}$ & Subtype ${ }^{3}$ & $\log M A R$ acuity ${ }^{4}$ & Refractive correction ${ }^{5}$ & Past treatment \\
\hline \multicolumn{10}{|c|}{ Neutral group } \\
\hline 1 & $\mathrm{~F}$ & 29 & L & 0.78 & s & s & 0.84 & $\mathrm{NaN}$ & patching, glasses, visual training for 2 yrs \\
\hline 2 & $\mathrm{~F}$ & 22 & R & 0.60 & s & M & 0.62 & $\begin{array}{l}\text { OD: }+3.00, \text { OS: }+2.00 \\
\text { contacts; OD: }+6.5-1.00\end{array}$ & patching \\
\hline 3 & $\mathrm{~F}$ & 21 & $\mathbf{R}$ & 0.80 & s & A & 0.72 & $\begin{array}{c}\times 160, \text { OS: }+2.00-0.25 \times \\
100\end{array}$ & patching, eyedrops, corrective lenses \\
\hline 4 & $\mathbf{F}$ & 27 & L & 0.60 & MO & M & 0.52 & $\begin{array}{l}\text { OD: }-5.00-0.75 \times 160 \\
\text { OS: }-7.5-3.75 \times 007\end{array}$ & patching, corrective surgery, corrective lenses \\
\hline 5 & M & 24 & $\mathbf{R}$ & 0.74 & MO & M & 0.54 & $\begin{array}{l}\text { OD: }+2.75-0.5 \times 105 \\
\text { OS: }-0.5-0.25 \times 175\end{array}$ & patching, corrective lenses \\
\hline 6 & M & 24 & R & 0.30 & MO & M & 0.32 & $\begin{array}{l}\text { OD: }+4.25-3.25 \times 179 \\
\text { OS: }+2.75-2.25 \times 009\end{array}$ & noncomplant patching, corrective lenses \\
\hline 7 & $\mathrm{~F}$ & 22 & L & 0.48 & MO & A & 0.46 & $\begin{array}{l}\text { OD: }-4.00-1.75 \times 160 \\
\text { OS: }-1.50-0.75 \times 180 \\
\text { contacts: OD }-7.25 \mathrm{BC}\end{array}$ & glasses \\
\hline 8 & M & 21 & L & 0.52 & MO & M & 0.56 & $\begin{array}{l}\text { 8.4 DIA 13.8, OS: }-6.75 \\
\text { BC } 8.4 \text { DIA } 13.8\end{array}$ & noncompliant patching, glasses \\
\hline 9 & $\mathrm{~F}$ & 21 & $R$ & 0.24 & MI & s & 0.18 & $\begin{array}{c}\text { contacts; OD: }+3.75 \text {, OS: } \\
+3.75\end{array}$ & patching, stereo training, corrective lenses \\
\hline 10 & $\mathrm{~F}$ & 19 & L & 0.40 & Mo & s & 0.52 & $\begin{array}{l}\text { glasses; OD: } 3.00, \mathrm{OS}:- \\
\quad 3.75\end{array}$ & $\begin{array}{l}\text { patching, eye strengthening exercises, corrective } \\
\text { lenses }\end{array}$ \\
\hline Totals & $\begin{array}{l}\text { M: } 3 \\
F: 7\end{array}$ & $M=23.0 \pm .966$ & $\begin{array}{l}\text { L: } 5 \\
\text { R: } 5\end{array}$ & $M=.546 \pm .062$ & $\begin{array}{c}\text { S: } 3 \\
\text { MO: } 6 \\
\text { MI: } 1\end{array}$ & $\begin{array}{l}S: 3 \\
A: 2 \\
M: 5\end{array}$ & $M=.528 \pm .059$ & & $\begin{array}{c}\text { patching: } 7 \\
\text { corrective surgery: } 1 \\
\text { corrective lenses: } 10 \\
\text { visual training exercises: } 3\end{array}$ \\
\hline \multicolumn{10}{|c|}{ Attention group } \\
\hline 1 & $\mathrm{~F}$ & 21 & L & 0.42 & мо & A & 0.54 & $\mathrm{NaN}$ & none \\
\hline 2 & $\mathrm{~F}$ & 20 & L & 0.60 & MO & $\mathrm{s}$ & 0.52 & $\mathrm{NaN}$ & patching \\
\hline 3 & $\mathrm{~F}$ & 22 & L & 1.02 & $\mathrm{~s}$ & M & 0.94 & $\mathrm{NaN}$ & patching \\
\hline 4 & F & 19 & R & 0.66 & MO & A & 0.54 & $\begin{array}{l}\text { contacts; OD: } 8.5, \mathrm{OS}:- \\
2.5\end{array}$ & none \\
\hline 5 & M & 22 & L & 0.56 & MO & s & 0.52 & $\mathrm{NaN}$ & patching, noncompliant eye drops, glasses \\
\hline 6 & M & 20 & $\mathbf{R}$ & 0.44 & MO & s & 0.36 & $\mathrm{NaN}$ & stereo training \\
\hline 7 & M & 33 & R & 0.22 & MI & M & 0.12 & $\begin{aligned} \text { OD: } & +3.5-2.5 \times 180, \text { OS: }: \\
& 4.00-1.5 \times 180\end{aligned}$ & patching, glasses \\
\hline 8 & M & 29 & L & 0.80 & s & A & 0.82 & $\mathrm{NaN}$ & noncompliant patch and eye strengthening exercises \\
\hline 9 & $\mathrm{~F}$ & 19 & L & 0.26 & MI & M & 0.22 & $\begin{array}{l}\text { OD: }-0.50-0.25 \times 62 \\
\text { OS: }+2.00\end{array}$ & noncompliant patching, refractive correction \\
\hline 10 & M & 37 & $\mathbf{R}$ & 0.46 & MO & s & 0.42 & $\begin{array}{l}\text { OD: }+3.75-1.25 \times 175 \\
\text { OS: }+3.00-1.50 \times 018\end{array}$ & patching, glasses \\
\hline Totals & $\begin{array}{l}\text { M: } 5 \\
\text { F: } 5\end{array}$ & $M=24.2 \pm 2.037$ & $\begin{array}{l}\text { L: } 6 \\
\text { R: } 4\end{array}$ & $M=.594 \pm .088$ & $\begin{array}{c}\text { S: } 2 \\
\text { MO: } 6 \\
\text { MI: } 2\end{array}$ & $\begin{array}{l}\text { S: } 4 \\
\text { A: } 3 \\
\text { M: } 3\end{array}$ & $M=.509 \pm .087$ & & $\begin{array}{c}\text { patching: } 6 \\
\text { corrective surgery: } 0 \\
\text { corrective lenses: } 7 \\
\text { visual training exercises: } 1\end{array}$ \\
\hline \multicolumn{10}{|c|}{ 'Depth = (LogMAR VA amblyopic eye - LogMAR VA fellow eye); as calculated in Popple \& Levi, 2008.} \\
\hline \multicolumn{10}{|c|}{${ }^{2}$ Amblyopic severity (according to amblyopic eye logMAR acuity; Stewart et al., 2005): MI = mid (0.1-0.3 logMar), MO = moderate $(>0.3-0.6 \log M a r), S=s e v e r e(>0.6 \log M a r)$} \\
\hline \multicolumn{10}{|c|}{${ }^{3} \mathrm{~S}=$ strabismic, $\mathrm{A}=$ anisometropic, $\mathrm{M}=$ mixed } \\
\hline \multicolumn{10}{|c|}{${ }^{4}$ Amblyopic eye logMAR acuity } \\
\hline
\end{tabular}

Table 1. Demographic and clinical information for all observers.

Phases I \& III: Pre-training \& Post-training. Day 1 consisted of a prescreening session in which we determined whether observers fulfilled our inclusion criteria (see Observers) and obtained a baseline measure of their abilities on a curated selection of visual tests known to be impaired in amblyopia: foveal broadband contrast sensitivity, logMar visual acuity, crowding, and stereoacuity (see Supplemental Information for details). After obtaining consent and having participants fill out a demographic survey, we conducted a full oral interview of their visual history. The protocols for days 1 and 17 were identical from this point on. We measured observers' logMar visual acuity by having them read an eye chart (see Apparatus \& setup) monocularly (fellow eye always first) with the other eye patched, from $2 \mathrm{~m}$ away in a consistently brightly lit room. All remaining tasks were administered in a darkened and sound-attenuated room. Observers used a forehead and chin rest to ensure head stabilization and a constant viewing distance. On average, the prescreening session on day 1 took 2 hours and the post-training session on day 17 took 1.5 hours. On days 2 
and 16, we estimated observers' foveal broadband contrast sensitivity in their fellow and amblyopic eyes separately.

For all tasks, if observers moved their eyes $\geq 1^{\circ}$ from the fixation cross, the text 'Please fixate' would appear onscreen and that trial would be replaced at the end of the block. To account for procedural learning, observers first performed a simple two-alternative forced-choice (2AFC) orientation discrimination task (2-cpd, $64 \%$ contrast, $3.2^{\circ}$-wide Gabor tilted $\pm 5^{\circ}$ from horizontal) at the fovea for $\geq 40$ trials. Next, they completed 1-2 practice blocks of 80 trials along each diagonal. Two $3.2^{\circ}$-wide 6 -cpd Gabors (64\% contrast) simultaneously appeared at $4^{\circ}$ eccentricity along one of the diagonals. The observer had to report the orientation $\left( \pm 4^{\circ}\right.$ from horizontal) of the target indicated by a central response cue.

On day 3 , observers completed an adaptive staircase procedure to estimate the monocular contrast thresholds required for each eye to perform the main training task with similar accuracy before training along the same diagonal to which they were randomly assigned to train (upper-right and lower-left quadrants or upper-left and lower-right quadrants). Observers completed four 40-trial blocks with their fellow eye (with amblyopic eye patched), and after a break, they completed four blocks with their amblyopic eye (with fellow eye patched). The contrast of the Gabors adjusted on each trial according to best PEST —an adaptive staircase procedure as implemented in the Palamedes toolbox [80] - to estimate contrast thresholds for $75 \%$ and $88 \%$ accuracy in two interleaved staircases. A uniform prior was used for the first block, and starting from the second block, the posterior of the previous block was used as the prior for the next. The threshold estimates from the last three blocks in each eye were averaged and used as the individual observer's contrast levels throughout the rest of the study. Observers were only presented with neutral attention cues (see Main task stimuli). On average this session took 1.5-2 hours.

Days 4 and 15 were the pre-test and post-test sessions for the main training task. Before running the main task in each eye, we conducted another staircase to assess whether contrast threshold estimates remained the same from the prior session. If there was a large discrepancy (perhaps due to some short-term consolidation of learning), the experimenter would manually adjust the stimulus contrast levels for each eye to better capture the dynamic range of the observer and make the task challenging enough that they would have plenty of room for improvement with training (i.e., performed with a d' around 1 overall in each eye). Stimulus contrast was then held constant throughout the remainder of the study. We used the individualized contrast thresholds for the amblyopic and fellow eyes to measure baseline performance on the main task in four conditions, in this order: (1) fellow eye along the upper-right and lower-left diagonal, (2) amblyopic eye along the upper-right and lower-left diagonal, (3) fellow eye along the upper-left and lower-right diagonal, and (4) amblyopic eye along the upper-left and lower-right diagonal. All observers were only exposed to neutral cues during the pre-test and post-test. On average these pre-test and post-test sessions took 1.5-2 hours.

Phase II: Training. Observers completed 6,400 trials total: approximately eight 80-trial blocks during each of $\sim 10$ training sessions. We hypothesized that this training protocol would be sufficient to result in significant VPL for the majority of observers with amblyopia, but not so much that it would promote specificity, as has been shown in neurotypical adults [81].

During each 1-hour training session, observers completed as many 80 -trial blocks of the main 2AFC orientation task (see Main task trial sequence) as possible using their amblyopic eye with their fellow eye patched. During training sessions, observers took 30 -second breaks halfway through each block (a neutral auditory tone indicated when they should open their eyes), paused between blocks, and had a 5-minute break halfway through to minimize oculomotor fatigue and retinal adaptation. Each observer was randomly assigned to train along one of the two diagonals 
(five observers trained on each diagonal within each group) while being exposed to only neutral cues (Neutral group; $n=10$ ) or $100 \%$ valid peripheral cues (Attention group; $n=10$ ). To maximize the effect of stimulating the trained diagonal, we used only valid peripheral cues, which automatically (requiring no additional effort or even awareness of the cue from the observer) direct attention to the location of the upcoming target, and not invalid cues, which direct attention to a nontarget location. Cue validity does not affect the magnitude of either the benefit or cost of exogenous attention on performance during classic spatial cueing experiments ([82]; review[25]).

Main task stimuli. Observers were asked to fixate on a black cross ( $1^{\circ}$ across) at the center of the screen throughout the trial (Figure 1a). The target and distractor stimuli were both $3.2^{\circ}$-wide, 6 -cpd Gabor patches (contrast-defined sinusoidal gratings embedded in a Gaussian envelope, $\sigma=0.46^{\circ}$ ), randomly and independently tilted $\pm 5^{\circ}$ from horizontal, centered at $4^{\circ}$ eccentricity along the diagonals, and with the same mean luminance as the uniform grey background $\left(26 \mathrm{~cd} / \mathrm{m}^{2}\right)$. The neutral precue consisted of four $0.16^{\circ}$ white dots surrounding the fixation cross and centered $0.5^{\circ}$ away from its edges. The peripheral exogenous attention precue was identical to the neutral cue but centered around and placed $0.5^{\circ}$ from the edge of the target in the periphery, to prevent masking. The response cue (a $0.8^{\circ}$-long white line starting $0.8^{\circ}$ from the center of the screen) indicated the target location by pointing to one stimulus location (matching the peripheral attention precue with $100 \%$ validity).

Main task trial sequence. Observers monocularly (with the other eye patched) performed a 2AFC orientation discrimination task while their exogenous spatial attention was manipulated via presentation of a brief valid peripheral precue (Figure 1a). After centrally fixating (256 ms), the precue was presented (67 ms), after which there was a brief interstimulus interval (ISI; $67 \mathrm{~ms}$ ) during which the screen was blank. The target and distractor Gabor stimuli then appeared at isoeccentric locations along one of the diagonals concurrently with the response cue for $123 \mathrm{~ms}$. This 134-ms stimulus-onset-asynchrony between the onset of the precue and onset of the stimuli enabled us to assess the attentional effects of the exogenous cue while preventing any voluntary deployment of attention (review[25]). After the Gabors disappeared, the response cue stayed throughout the remainder of the trial. 78-ms after the stimuli were removed, the fixation cross turned white and an auditory tone indicated the beginning of the response window, in which observers had to report the target orientation (counterclockwise or clockwise relative to horizontal) using one of two keyboard presses (' 1 ' for counterclockwise, '2' for clockwise) with their right hand. On every trial, observers were encouraged to respond as accurately as possible within a 5-s response window. Observer response terminated the trial, after which there was a mandatory 1000-ms intertrial interval. Auditory feedback was provided at the end of each trial and visual feedback indicating observers' accuracy and number of fixation breaks was presented at the end of each block.

Acknowledgments: This research was supported by National Eye Institute RO1-EY027401 to M.C., National Eye Institute T32-EY007136 to New York University, National Science Foundation Division of Graduate Education DGE-1342536 to M.R., and the New York University Graduate School of Arts \& Sciences Dean's Dissertation Fellowship to M.R. We thank Julia Payne and Sanjana Manjunath for their help with data collection, Antonio Fernández, Shao-Chin Hung, Michael Jigo, Caroline Myers, Angela Shen and other members of the Carrasco Lab, as well as Sarit Szpiro, Amit Yashar, Roozbeh Kiani, Lynne Kiorpes, Zhong-Ling Lu and Denis Pelli for helpful comments.

Author Contributions: M.C. and M.R. designed the experiments, interpreted the data and wrote the manuscript. M.R. programmed the experiment and collected and analyzed the data. 


\section{SUPPLEMENTAL INFORMATION}

Main task performance. We conducted a 4-way mixed ANOVA on d' for all observers (Figure S1, top row) with factors of Session (pre-test, post-test), Eye (amblyopic, fellow), Diagonal (trained, untrained) and Group (Neutral, Attention). There were significant main effects of Session $\left(F(1,18)=5.97, p=.025, \eta^{2} G=.058\right)$ and Diagonal $\left(F(1,18)=5.55, p=.030, \eta^{2} G=.018\right)$, and a 3-way interaction among Session, Diagonal, and Group $\left(F(1,18)=4.39, p=.050, \eta^{2} G=.006\right)$. Neither the main effects of Eye or Group nor all other interactions were significant (all $p>0.3$ ). The complementary 3-way (Eye X Diagonal X Group) mixed ANOVA of normalized proportion change revealed a significant 2-way interaction between Diagonal and Group $(F(1,18)=7.00, p=.016$, $\eta^{2} G=.042$ ); the Neutral group exhibited significantly more improvement at the trained than untrained diagonal $(t(9)=3.19, p=.011,95 \% \mathrm{Cl}=[.044, .260])$, whereas the Attention group improved to a similar extent at both diagonals $(t(9)=-.031, p=.976,95 \% \mathrm{Cl}[-.146, .142])$. None of the main effects or other interactions were significant (all $p>1$ ).

A corresponding analysis of median RT, our secondary variable, showed a significant main effect of Session $\left(F(1,18)=28.4, p<.001, \eta^{2} G=.112\right)$, but no other significant main effects or interaction (all p>.2; Figure S1, bottom row). The complementary 3-way (Eye X Diagonal X Group) mixed ANOVA of normalized proportion change indicated a significant Eye $x$ Diagonal interaction $(F(1,18)=4.88$, $\left.p<.05, \eta^{2} G=.010\right)$, but post-hoc t-tests found no significant differences across all diagonals in both eyes (all $p>$.2). All other main effects and interactions were not significant (all $p>.1$ ).

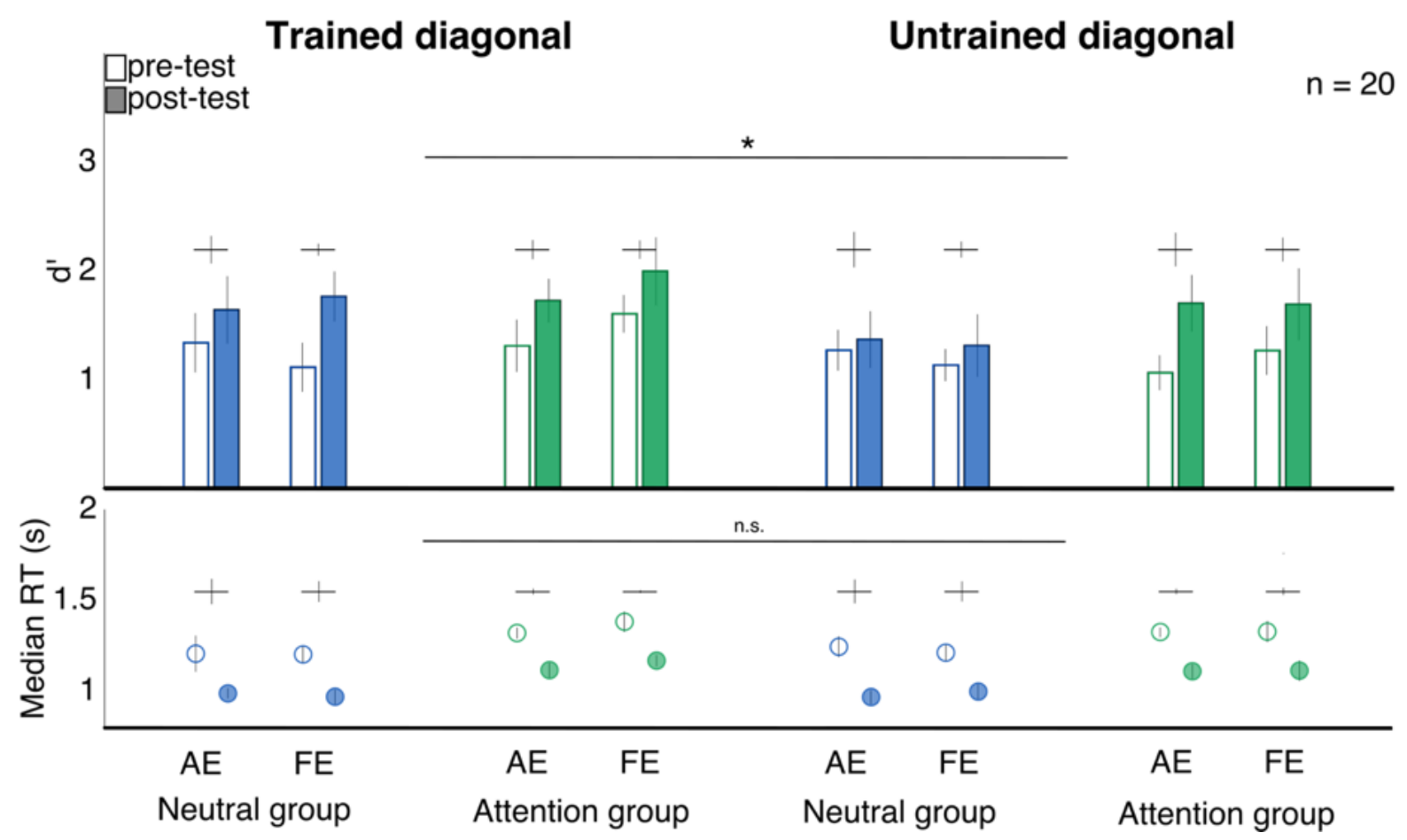

Figure S1, related to Figure 2. Performance for all observers on the main training task at pre-test and posttest, split by diagonal, group and eye. $\mathrm{AE}=$ amblyopic eye; $\mathrm{FE}=$ fellow eye. Error bars represent \pm 1 withinsubject standard error [53]. * $p<.05$. 
qCSF. The neural and behavioral markers of amblyopia are present across the visual field, including the parafovea, where our training stimuli were placed (e.g.[14-18)]. However, amblyopia has typically been characterized as a foveal visual disorder (e.g.[13]). Therefore, we also measured broadband contrast sensitivity at the center of observers' gaze on days 2 and 16 (before and after training) using the qCSF, or quick Contrast Sensitivity Function method. The qCSF is a Bayesian adaptive procedure that uses a trial-by trial information gain strategy to estimate and detect changes in the underlying contrast sensitivity functions of neurotypical (e.g.[83]) and special populations, including adults with amblyopia $[84,85]$.

We incorporated the qCSF into a 4AFC orientation discrimination task (Figure S2, left side). The $5.6^{\circ}$-wide target stimulus could be any combination of parameters from a $2 \mathrm{D}$ array of spatial frequencies (12 levels ranging from 0.25 - to 20 -cpd) and contrasts (60 levels ranging from $1 \%$ to $100 \%$; Figure S2, right side). After at least one practice block in which they binocularly performed the task above chance using an easier (higher contrast range) stimulus set, observers completed three 70-trial blocks with each eye while the other was patched. Observers always started with their fellow eye and alternated eyes every block. If observers moved their eyes more than $1^{\circ}$ from the fixation cross, the text 'Please fixate' would appear onscreen and that trial would be cancelled and added to the end of the block. On average the qCSF session took $75 \mathrm{~min}$.

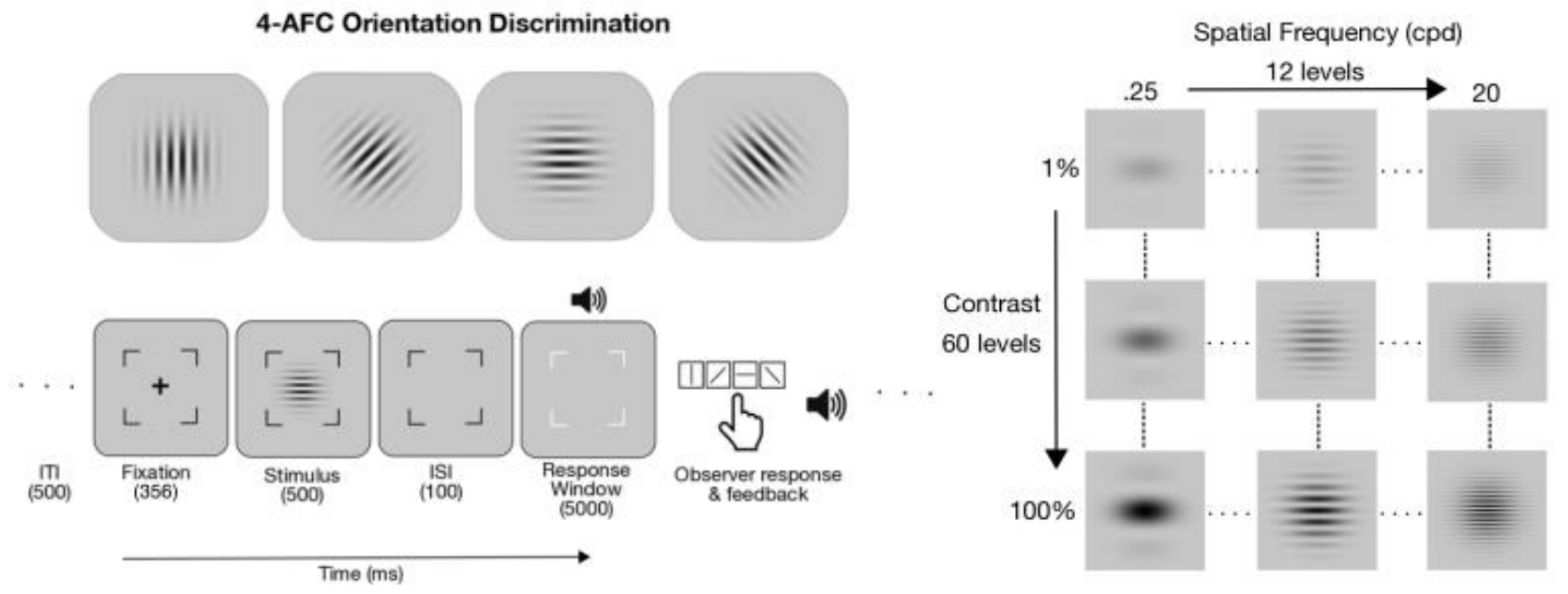

Figure S2, related to STAR Methods. The trial sequence for the qCSF behavioral task (left), and 2D array of all possible conjunctions of spatial frequency and contrast for the target stimulus (right).

Each observer's CSF was modeled as a truncated log-parabola with four parameters: peak sensitivity, peak spatial frequency, bandwidth, and truncation. Each model parameter was treated as a separate dependent variable in a series of 3-way (Eye: amblyopic, fellow X Group: Neutral, Attention X Session: pre-test, post-test) mixed ANOVAs (Figure S3a). The main effect of Eye was always significant (amblyopic eye<fellow eye; all $p<.015$ ). There was a significant 2-way interaction between Group and Eye for the truncation parameter $\left(F(1,17)=5.24, p=.035, \eta^{2}{ }_{G}=.028\right)$ because the Neutral group showed a significant difference between the two eyes $(t(9)=-4.81, p<.001,95 \%$ $\mathrm{Cl}[-.187,-.067])$, but the Attention group did not $(t(8)=-.893, p=.398,95 \mathrm{Cl} \%[-.095, .042])$. There was also a significant 2-way interaction between Session and Eye for peak sensitivity $\left(F(1,17)=8.57, p=.009, \eta^{2} G=.010\right)$; whereas there was a significant change in peak sensitivity from pre-test to post-test in the amblyopic eye $(t(18)=-2.81, p=.012,95 \mathrm{Cl} \%[-.132,-.019])$, there was not in the fellow $(t(18)=-.775, p=.449,95 \mathrm{Cl} \%[-.074, .034])$. All other 2- and 3-way interactions were not significant (all $p>$.3). Complementary 2-way (Eye: amblyopic, fellow X Group: Neutral, Attention) ANOVAs of the normalized proportion change (Figure $\mathrm{S} 3 \mathrm{~b}$ ) revealed a significant main effect of 
Eye for peak sensitivity $\left(F(1,17)=7.68, p=.013, \eta^{2} G=.057\right.$; amblyopic eye $>$ fellow eye $)$ and peak spatial frequency $\left(F(1,17)=7.79, p=.013, \eta^{2} G=.171\right.$; fellow eye >amblyopic eye), but no main effect of Group or 2-way interactions (all $p>.2$ ).

The results suggest that contrast sensitivity gains were largely driven by changes in peak sensitivity, rather than peak spatial frequency, or increased sensitivity to lower or higher spatial frequencies. One VPL study in which amblyopic observers showed increased sensitivity to higher spatial frequencies had observers train on a contrast sensitivity task near their individual SF cutoffs [86]. We note that our observers trained on a 6-cpd Gabor, a challenging spatial frequency for amblyopes but not close to their spatial frequency cutoff.

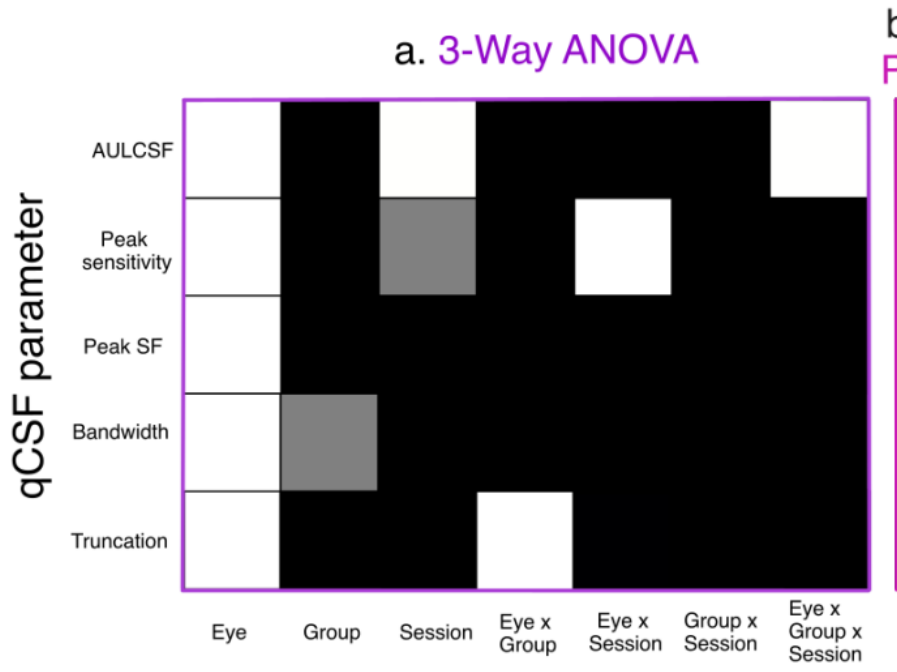

\section{b. 2-Way ANOVA:} Proportion change

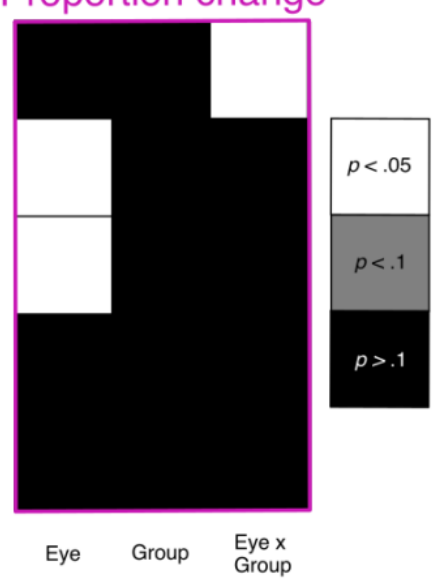

Figure S3a, related to Figure 5. A graphical representation of the results of a series of 3-way mixed ANOVAs for all qCSF parameter values (left table) and b. 2-WAY ANOVAs for their corresponding normalized proportion change values (right table). The shading of each square represents the statistical significance of the main effect or interaction in each of the respective tasks.

A Pearson's correlation matrix analysis revealed that, aside from the truncation parameter that did not correlate with any of the other parameters (all $p>.05$ ) and a nonsignificant correlation between peak gain and bandwidth ( $p=.124)$, all of the qCSF parameters significantly covaried with each other (all $p<.05$ ) and with a single value that effectively captures simultaneous changes in all model parameters, the 'area under the log qCSF' (AULCSF; all correlations $p<.001$ ). The AULCSF is a reliable and comprehensive index of a neurotypical (e.g.[83]) or amblyopic [84,85] observer's 'window of visibility' as it estimates contrast sensitivity across the wide range of spatial frequencies that compose most visual scenes; therefore, a higher value indicates greater contrast sensitivity.

A 3-way (Eye: amblyopic, fellow X Group: Neutral, Attention X Session: pre-test, post-test) mixed ANOVA (Figure 5a) of observers' individual AULCSF values (Figure S4) revealed significant main effects of Eye $\left(F(1,17)=12.3, p=.003, \eta^{2} G=.203\right.$; amblyopic eye $M=1.23 \pm .052$, fellow eye $M=1.50 \pm .051)$, Session $\left(F(1,17)=4.58, p=.047, \eta^{2} G=.037\right.$; pre-test $M=1.32 \pm .017$, post-test $M=1.42 \pm .045)$, and a 3-way interaction of Eye, Group, and Session $\left(F(1,17)=5.84, p=.027, \eta^{2} G\right.$ $=.007)$. A 2-way ANOVA within the Neutral group revealed a significant Eye $X$ Session interaction $\left(F(1,9)=9.51, p=.013, \eta^{2} G=.010\right)$, with greater pre-test to post-test improvement in the amblyopic eye $(t(9)=2.16, p=.060,95 \% \mathrm{Cl}[-.006, .234])$ than fellow eye $(t(9)=.136, p=.895,95 \% \mathrm{Cl}[-$ $.094, .106])$. A 2-way ANOVA within the Attention group only revealed a main effect of Eye 
$\left(F(1,9)=6.66, p=.030, \eta^{2} G=.229\right)$; the main effects of Session and interaction were not significant (both $p>$.1). The complementary 2-way (Eye: amblyopic, fellow X Group: Neutral, Attention) mixed ANOVA of normalized proportion change in the AULCSF (Figure 5b) only revealed a significant 2way interaction of Eye and Group $\left(F(1,17)=4.67, p=.045, \eta^{2} G=.045\right)$; the amblyopic eye

$(M=.068 \pm .028)$ improved more than the fellow eye $(M=-.002 \pm .028)$ in the Neutral group $(t(9)=2.51$, $p=.033,95 \% \mathrm{Cl}[.007, .133])$, but the improvement was similar in the amblyopic eye $(M=.058 \pm .037)$ and fellow eye $(M=.068 \pm .037)$ in the Attention group $(t(8)=-.263, p=.799,95 \% \mathrm{Cl}[-.095, .075])$.
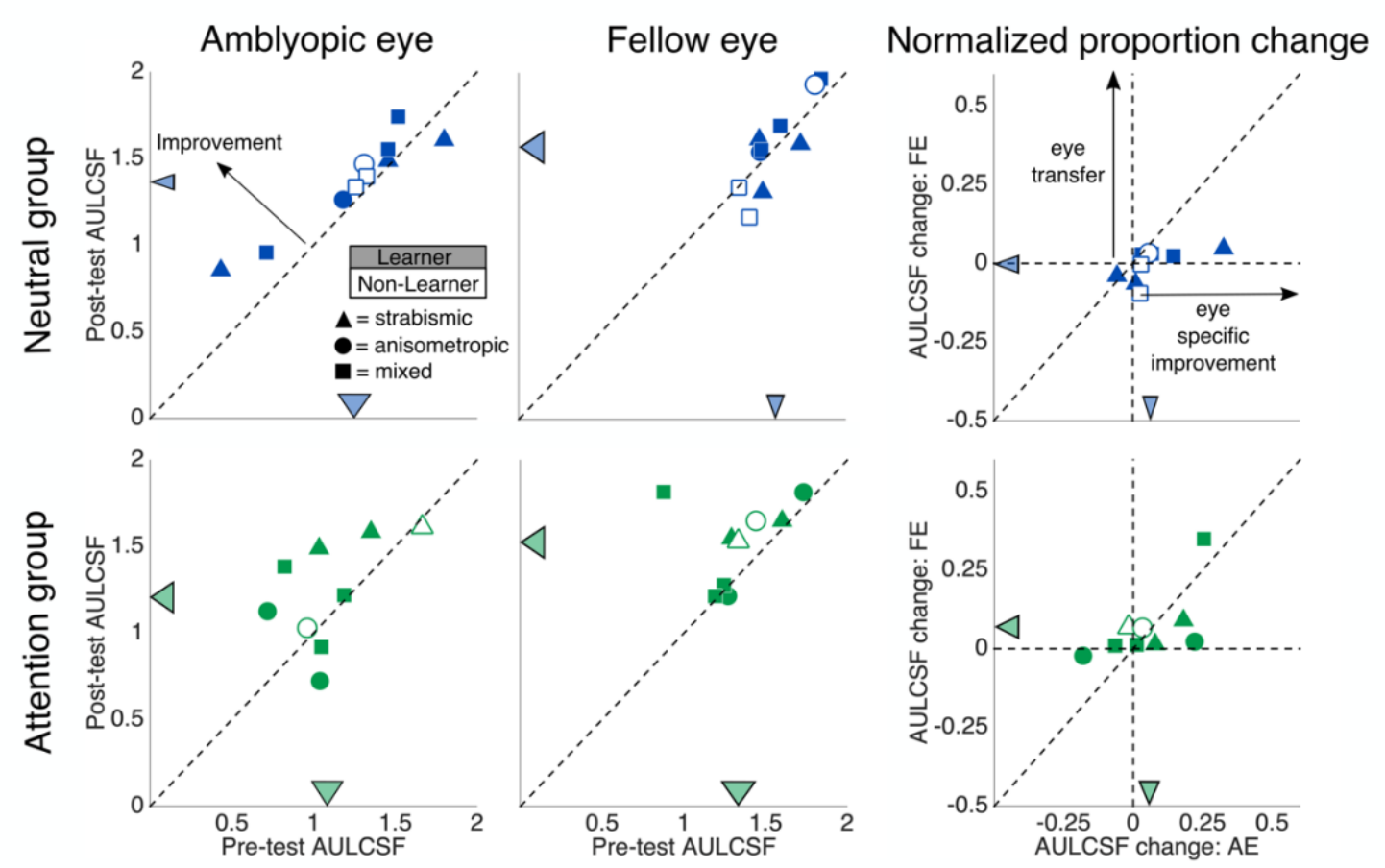

Figure S4, related to Figure 5. AULCSF values on the qCSF task for 10 observers in the Neutral (top row) and 9 observers in the Attention group (bottom row) at post-test versus pre-test for the amblyopic eye (left column), fellow eye (middle column), and the relative normalized proportion change in each (right column). The crossed lines symbolize group means, and their lengths indicate \pm 1 within-groups SEM [53].

Thus, both groups showed modest increases in the AULCSF, indicative of broadband improvements in foveal contrast sensitivity. The Attention group showed AULCSF gains in both eyes, whereas the Neutral group only showed gains in the amblyopic eye (although it is possible that a ceiling effect limited our ability to detect any changes in the fellow eye).

Pelli-Robson contrast sensitivity. We measured Pelli-Robson scores as a convergent, but relatively coarse, measure of observers' broadband contrast sensitivity at the fovea (Figure S5 for individual scatter plots). Log contrast sensitivity thresholds for letters were measured using one of two versions of the Pelli-Robson Contrast Sensitivity Chart [87]. Participants monocularly (with the other eye patched) read one version with their fellow eye and then the other version with their amblyopic eye. Pelli-Robson scores were graded as the log contrast sensitivity threshold corresponding to the last set of letter triplets in which they identified two letters correctly, reading from top left to bottom right [87], with higher scores indicating greater contrast sensitivity. A 3-way (Eye: amblyopic, fellow X Group: Neutral, Attention X Session: pre-test, post-test) mixed ANOVA (Figure 5a) revealed significant main effects of Eye $\left(F(1,17)=4.66, p=.045, \eta^{2} G=.078\right.$; amblyopic eye $M=1.77 \pm .039 \log$ contrast threshold, fellow eye $M=1.89 \pm .035 \mathrm{log}$ contrast threshold), Session $\left(F(1,17)=7.92, p=.012, \eta^{2} G=.050\right.$; pre-test $M=1.79 \pm .037$ log contrast threshold, 
post-test $M=1.87 \pm .027$ log contrast threshold), and a 2-way interaction of Eye and Session $\left(F(1,17)=4.51, p=.049, \eta^{2} G=.028\right)$, as there was a significant change between sessions in the amblyopic eye $(t(18)=-3.63, p=.002,95 \% \mathrm{Cl}[-.237,-.063]$, but not the fellow eye $(t(18)=-.383$, $p=.706,95 \% \mathrm{Cl}[-.102, .071])$. The main effect of Group, all other 2-way interactions, and the 3-way interaction were not significant (all $p>$.1). The 2-way (Eye: amblyopic, fellow X Group: Neutral, Attention) mixed ANOVA of the normalized proportion change (Figure $5 \mathrm{~b}$ ) revealed only a significant main effect of Eye $\left(F(1,17)=6.27, p=.023, \eta^{2} G=.148\right.$; amblyopic eye $M=.045 \pm .012$, fellow eye $M=.004 \pm .011$ ); all other main effects and interactions were not significant (all $p>.1$ ).

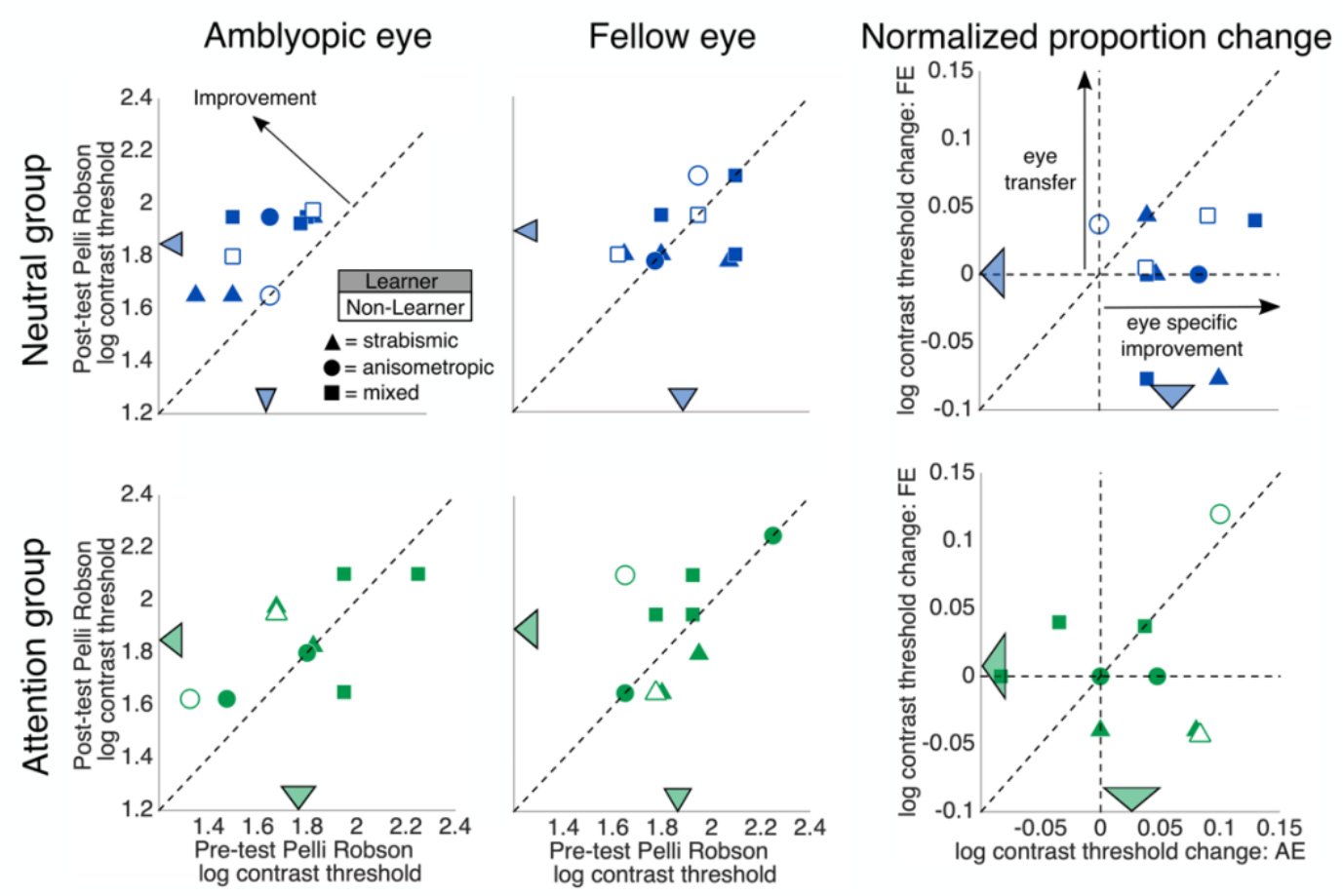

Figure S5, related to Figure 5. Log contrast thresholds for letters on the Pelli-Robson chart for 10 observers in the Neutral (top row) and 9 observers in the Attention group (bottom row) at post-test versus pre-test for the amblyopic eye (left column), fellow eye (middle column), and the relative normalized proportion change in each (right column). The crossed lines symbolize group means, and their lengths indicate \pm 1 within-groups SEM [53].

In summary, our two measures of foveal broadband contrast sensitivity showed partial agreement: the more sensitive measure, the AULCSF, revealed that the Neutral group showed greater improvement in the amblyopic eye compared to the fellow eye and the Attention group showed similarly modest improvements in both eyes; whereas the coarser measure, Pelli Robson CS, revealed that both groups only showed a similar improvement in amblyopic eye contrast sensitivity at post-test compared to pre-test.

Critical spacing of crowding. We measured observers' critical spacing (degrees of visual angle required to reliably and accurately discriminate a central number from two flanking distractor numbers to the left and right) at the fovea using the Pelli Critical Spacing toolbox [88] for MATLAB. The task was presented on a 13-inch 2017 MacBook Air using MacOS Mojave in a dark and sound-attenuated room, with observers' heads stabilized by a forehead and chin rest. The stimulus display screen was flipped, then presented onto a mirror $114 \mathrm{~cm}$ away from the observer to simulate presenting the task at $228 \mathrm{~cm}$, the necessary distance for displaying letters of a sufficient 
range of sizes to measure small thresholds. The experimenter input the observer's verbal responses manually using an external keyboard attached to the laptop. After binocularly performing above chance in a 10-trial practice block, observers completed a 20-trial adaptive staircase three times in a row with each eye (with the other eye patched). We averaged the estimates from all three staircases to separately estimate the critical spacing of crowding for each eye.

A 3-way (Eye: amblyopic, fellow X Group: Neutral, Attention X Session: pre-test, post-test) mixed ANOVA of observers' critical spacing (Figure 5a; Figure S6 for individual scatter plots; higher values are worse) only revealed a significant main effect of Eye $\left(F(1,17)=11.5, p=.003, \eta^{2} G=.245\right.$; amblyopic eye $M=.374 \pm .076^{\circ}$, fellow eye $\left.M=.112 \pm .007^{\circ}\right)$. In contrast to all other tasks, there was not a significant main effect of Session $\left(F(1,17)=.770, p=.392, \eta^{2} G=.002\right.$; pre-test $M=.233 \pm .032^{\circ}$, post-test $M=.253 \pm .045^{\circ}$ ). All other main effects and interactions were not significant (all $p>.3$ ). Consistently, none of the main effects or interactions in the 2-way mixed ANOVA of normalized proportion change were significant (all $p>.7$; Figure 5b).
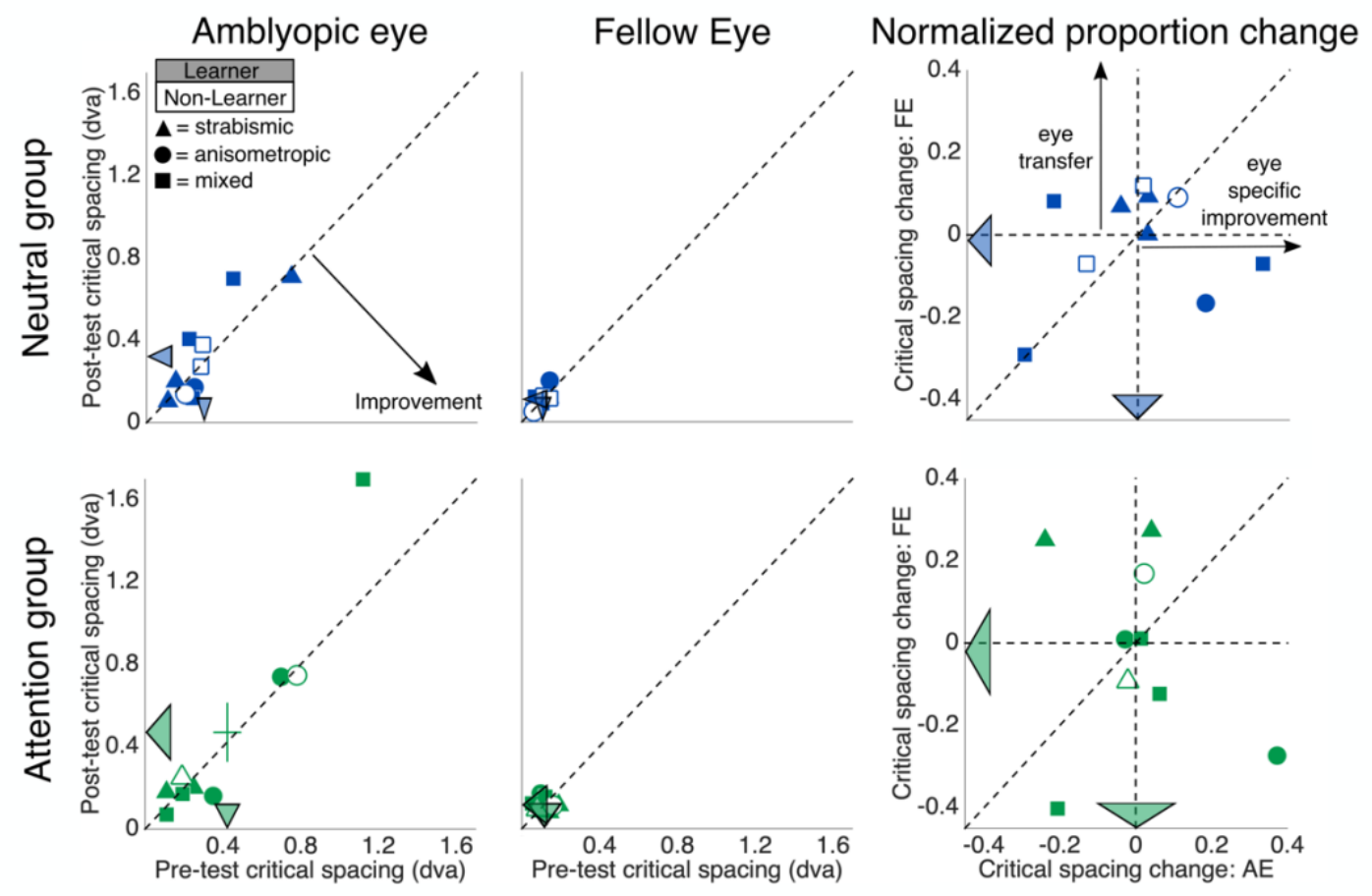

Figure S6, related to Figure 5. Critical spacing of crowding for 10 observers in the Neutral (top row) and 9 observers in the Attention group (bottom row) at post-test versus pre-test for the amblyopic eye (left column), fellow eye (middle column), and the relative normalized proportion change in each (right column). The crossed lines symbolize group means, and their lengths indicate \pm 1 within-groups SEM [53].

No significant changes in critical spacing of crowding. In contrast to the other tasks, the critical spacing of crowding did not reliably change in either eye of both groups. This is important as it shows that not all tasks improved after practice. This helps rule out that improvements on other tasks were simply due to procedural or motor learning, in which observers improve because they get more comfortable with the motor demands of the task, rather than attaining the longer-lasting changes in perceptual sensitivity or discriminability that define VPL (review[1]). Crowding and visual acuity have been shown to be weakly linked [89]; some observers may suffer from worse crowding but have near normal acuity, and vice versa [90]. Thus, improvements in visual acuity 
after training do not inherently predict simultaneous changes in crowding. Crowding in the fovea of neurotypical adults can be explained by simple pattern masking, but the mechanisms underlying crowding in amblyopic foveal vision may be more similar to crowding in the periphery of neurotypical adults, which has been proposed to result from an extended pooling stage following the feature detection stage [91]. There is preliminary evidence [92] that critical spacing is indicative of neural density (active neurons per square degree). Thus, sparse neural density may underly amblyopic crowding deficits, and may help explain why we did not see changes. Only ten days of training are unlikely to stimulate new neural growth and increase neural density. Although there are some perceptual learning studies with amblyopes showing that crowding can be reduced after practicing on a crowding task (e.g.[93]), training on our contrast sensitivity task did not transfer to crowding.

Stereoacuity thresholds. Stereoacuity thresholds were measured using the ASTEROID software program [94]. The task was presented on an Android 3D stereotablet presented at eye-level and parallel to the observer's head $25 \mathrm{~cm}$ away, held stable by a tablet stand whose base was clamped to the table. Observers performed the task in a darkened and sound-attenuated room with their head in a forehead and chin rest to ensure head stabilization. Observers were instructed to keep both eyes open and detect an illusory 3D square among four possible locations, then tap the corresponding quadrant of the screen. Observers first performed a short practice block of 20 trials, then completed two different adaptive Bayesian staircase blocks of 60 trials each. We averaged these two blocks to estimate an observer's stereoacuity threshold.

Given that stereoacuity is a binocular measure of depth perception, Eye was not included as a factor in a 2-way mixed ANOVA of stereoacuity thresholds (Figure 5a; Figure S7 for individual scatter plots; higher thresholds are worse). There was a significant main effect of Session $(F(1$, 17) $=5.60, p=.030, \eta^{2} G=.050$; pre-test $M=826 \pm 98.5$ arcsec, post-test $M=658 \pm 107$ arcsec), but the main effect of Group and 2-way interaction were not significant (both $p>.5$ ). A one-way ANOVA of the normalized proportion change in stereoacuity revealed that the between-subjects factor of Group was not significant ( $p>.4$; Figure $5 b)$.

Improvements in stereoacuity. Stereoacuity thresholds, or the distance in arcseconds required to reliably distinguish two objects as being at different planes of depth, are indicative of an observer's $3 \mathrm{D}$ vision. Amblyopia is most commonly a unilateral visual disorder (reviews[11,12]), and the clinical definition implies unequal weighting and improper integration of processing visual input from both eyes. Behaviorally, adults with amblyopia are often stereoblind, and suffer dramatic problems with depth during motor tasks from an early age (reviews[10,12]). Given that observers monocularly trained with the fellow eye patched, we were somewhat surprised to see that, as a whole, stereoacuity thresholds improved in both groups. This was particularly exciting as it suggests changes to observers' binocular vision even when not wearing an eye patch. Other PL studies in amblyopia have found incidental improvements in stereoacuity (e.g.[73]; reviews[12,22,23]). Some studies showing recovery of stereopsis and reduction in contrast sensitivity and acuity interocular disparities have used dichoptic perceptual training displays (reviews[9,10,12,22]). Classic 'push-pull' training protocols involve simultaneously "exciting" the under responsive amblyopic eye with transient cues which actively inhibit the overbearing fellow eye, in attempts to restore the proper interocular weighting of visual processing for each eye (reviews[10,95]). 


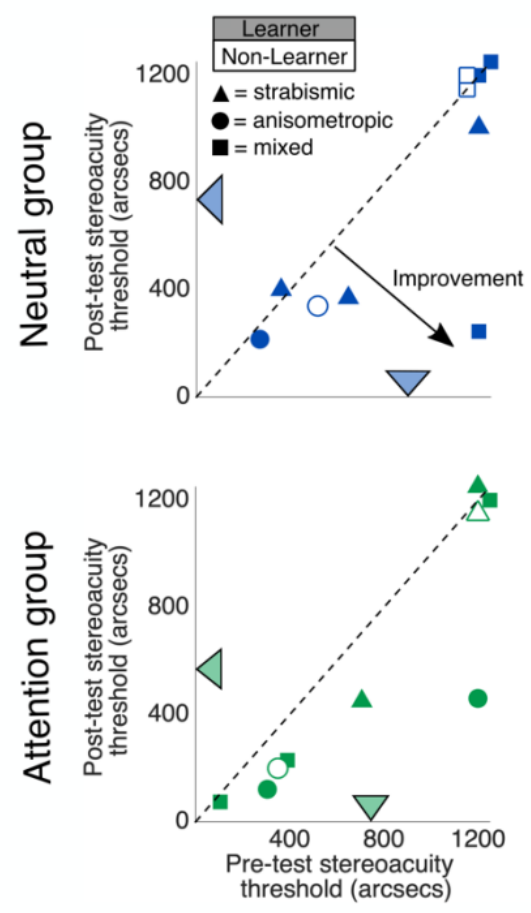

Figure S7, related to Figure 5. Stereoacuity thresholds for 10 observers in the Neutral (top row) and 9 observers in the Attention group (bottom row) at post-test versus pre-test. The crossed lines symbolize group means, and their lengths indicate \pm 1 within-groups SEM [53].

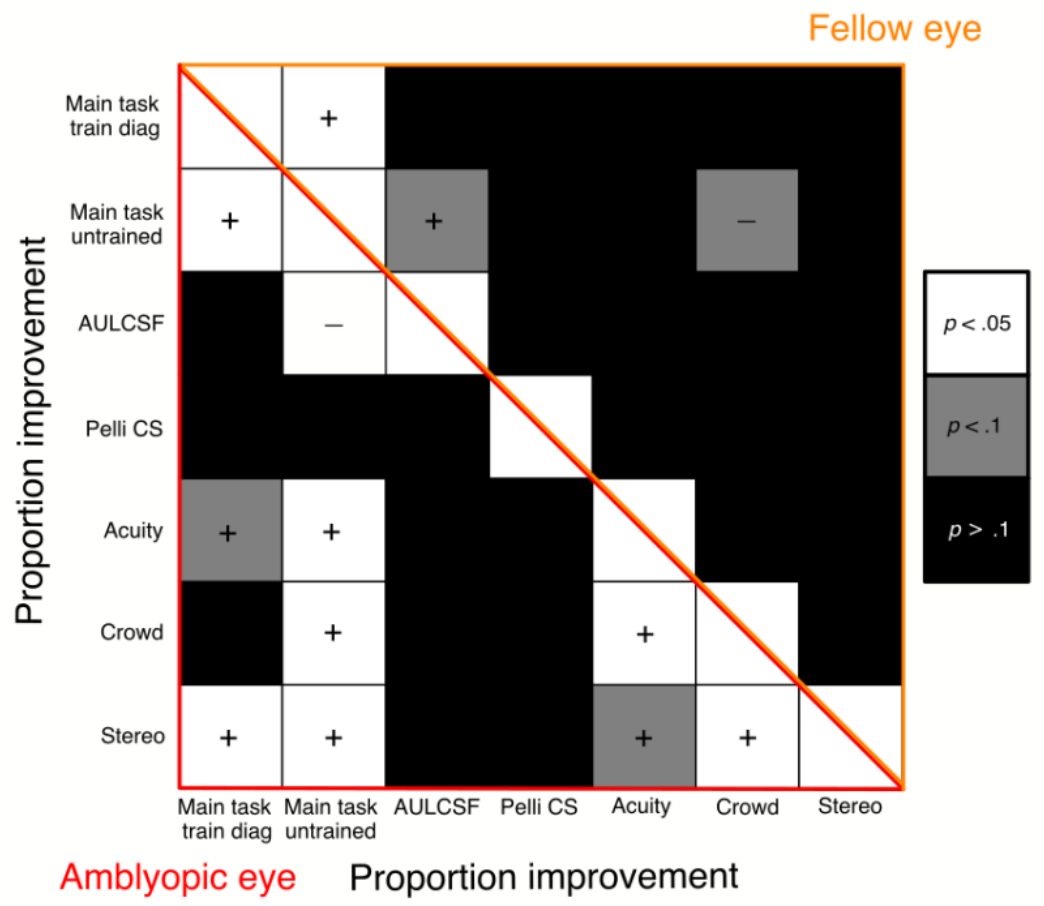

Figure S8, related to Figure 2. Correlation matrices of normalized proportion change across all tasks for the amblyopic eye (lower left triangle) and fellow eye (upper right triangle). The shading of each square represents the statistical significance of the correlation between the normalized proportion change in each of the respective tasks. A (+) symbol indicates that greater proportion improvement in one task predicts greater proportion improvement in the other, and (-) indicates that greater improvement in one task predicts smaller improvement in the other. 


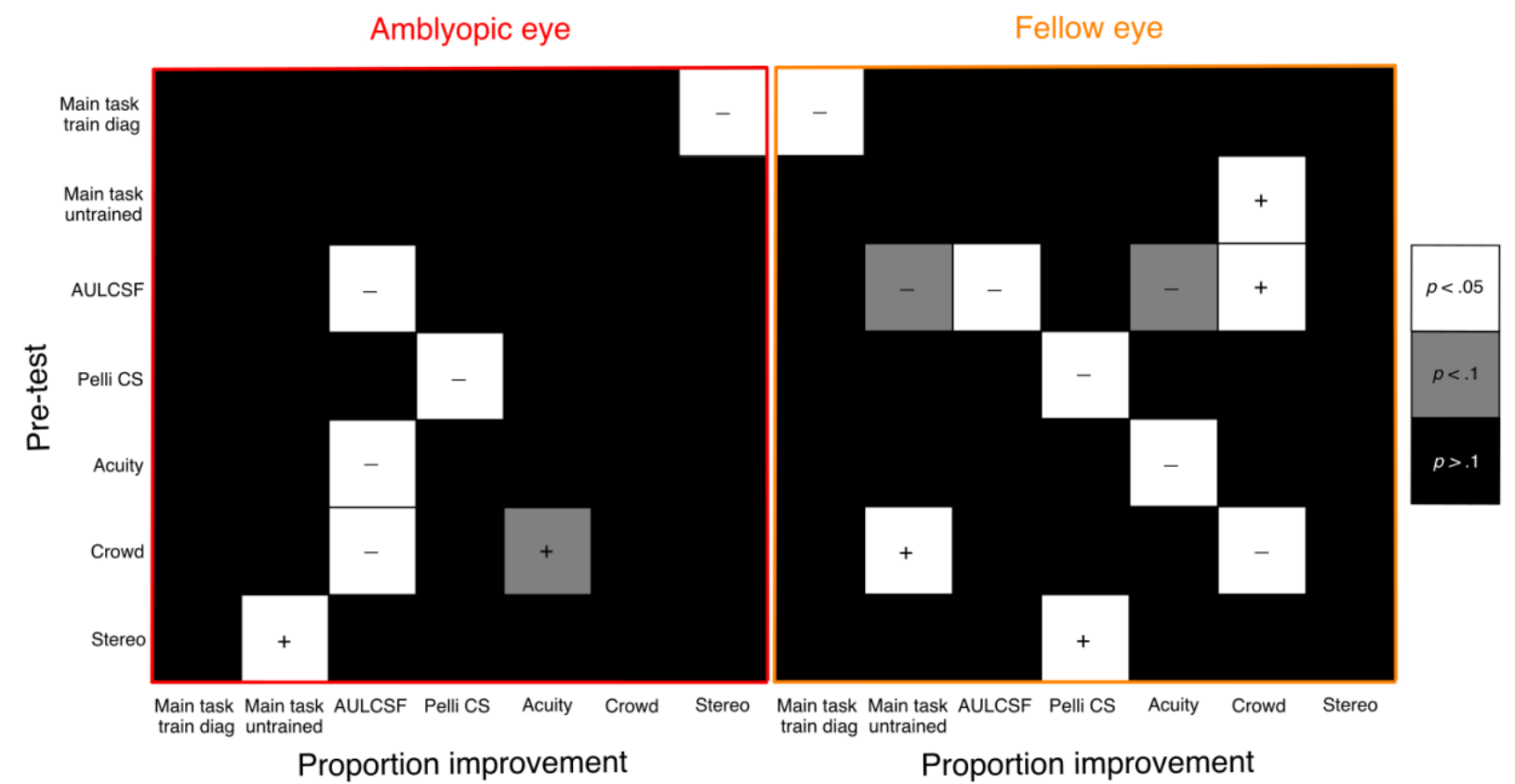

Figure S9, related to Figure 2. Correlation matrices of pre-test versus normalized proportion change in performance across all tasks for the amblyopic eye (left) and fellow eye (right). The shading of each square represents the statistical significance of the correlation between the pre-test value and normalized proportion change in each of the respective tasks. A (+) symbol indicates that a better value at pre-test predicted greater proportion improvement, while a (-) indicates that a better value at pre-test predicted a smaller proportion of improvement. 


\section{REFERENCES}

1. D. Sagi, Perceptual learning in Vision Research. Vision Res 51, 1552-1566 (2011).

2. C. D. Gilbert, W. Li, Adult visual cortical plasticity. Neuron 75, 250-264 (2012).

3. B. Dosher, Z.-L. Lu, Visual perceptual learning and models. Ann Rev Vis Sci 3, 343-363 (2017).

4. A. R. Seitz, Perceptual learning. Curr Biol 27, R631-R636 (2017).

5. M. Maniglia, A. R. Seitz, Towards a whole brain model of Perceptual Learning. Curr Opin Behav Sci 20, 47-55 (2018).

6. S. C. Hung, A. R. Seitz, Prolonged training at threshold promotes robust retinotopic specificity in perceptual learning. J Neurosci 34, 8423-8431 (2014).

7. R. Wang, J. Y. Zhang, S. A. Klein, D. M. Levi, C. Yu, Vernier perceptual learning transfers to completely untrained retinal locations after double training: a "piggybacking" effect. J Vis 14, 12-12 (2014).

8. S. F. Szpiro, B. A. Wright, M. Carrasco, Learning one task by interleaving practice with another task. Vision Res 101, 118-124 (2014).

9. F. Sengpiel, Plasticity of the visual cortex and treatment of amblyopia. Curr Biol 24, R936940 (2014).

10. D. M. Levi, D. C. Knill, D. Bavelier, Stereopsis and amblyopia: A mini-review. Vision Res 114, 17-30 (2015).

11. L. Kiorpes, N. Daw, Cortical correlates of amblyopia. Vis Neurosci 35, (2018).

12. D. M. Levi, Rethinking amblyopia 2020. Vision Res 176, 118-129 (2020).

13. C. Shooner et al., Population representation of visual information in areas V1 and V2 of amblyopic macaques. Vision Res 114, 56-67 (2015).

14. L. M. Katz, D. M. Levi, H. E. Bedell, Central and peripheral contrast sensitivity in amblyopia with varying field size. Doc Ophthalmol 58, 351-373 (1984).

15. C. Hou, Y. J. Kim, X. J. Lai, P. Verghese, Degraded attentional modulation of cortical neural populations in strabismic amblyopia. J Vis 16, 16-16 (2016).

16. M. Roberts, R. Cymerman, R. T. Smith, L. Kiorpes, M. Carrasco, Covert spatial attention is functionally intact in amblyopic human adults. J Vis 16, 1-19 (2016).

17. A. Pham, M. Carrasco, L. Kiorpes, Endogenous attention improves perception in amblyopic macaques. J Vis 18, 11-11 (2018).

18. P. V. Ramesh, M. A. Steele, L. Kiorpes, Attention in visually typical and amblyopic children. $J$ Vis 20, 11-11 (2020). 
19. K. Meier, D. Giaschi, Unilateral amblyopia affects two eyes: fellow eye deficits in amblyopia. Investig Ophthalmol Vis Sci 58, 1779-1800 (2017).

20. A. T. Astle, B. S. Webb, P. V. McGraw, Can perceptual learning be used to treat amblyopia beyond the critical period of visual development? Ophthalmic Physiol Opt 31, 564-573 (2011).

21. I. Tsirlin, L. Colpa, H. C. Goltz, A. M. Wong, Behavioral training as new treatment for adult amblyopia: A meta-analysis and systematic review. Invest Ophthalmol Vis Sci 56, 40614075 (2015).

22. A. Rodán, E. C. Marroquín, L. C. J. García, An updated review about perceptual learning as a treatment for amblyopia. J Optom, (2020). https://doi.org/10.1016/j.optom.2020.08.002

23. D. M. Levi, R. W. Li, Perceptual learning as a potential treatment for amblyopia: a minireview. Vision Res 49, 2535-2549 (2009).

24. M. I. Posner, Orienting of attention. Q J Exp Psycho/ 32, 3-25 (1980).

25. M. Carrasco, Visual attention: The past 25 years. Vision Res 51, 1484-1525 (2011).

26. M. Carrasco, A. Barbot, How attention affects spatial resolution. Cold Spring Harbor Symp Quant Biol 79, 149-160 (2015).

27. S. Szpiro, M. Carrasco, Exogenous attention enables perceptual learning. Psychol Sci 26, 1854-1862 (2015).

28. I. Donovan, S. Szpiro, M. Carrasco, Exogenous attention facilitates location transfer of perceptual learning. $J$ Vis 15, 11-11 (2015).

29. I. Donovan, M. Carrasco, Endogenous spatial attention during perceptual learning facilitates location transfer. J Vis 18, 7-7 (2018).

30. I. Donovan, A. Shen, C. Tortarolo, A. Barbot, M. Carrasco, Exogenous attention facilitates perceptual learning in visual acuity to untrained stimulus locations and features. J Vis 20, 18-18 (2020).

31. K. N. Nguyen, T. Watanabe, \& G. J. Andersen, Role of endogenous and exogenous attention in task-relevant visual perceptual learning. Plos one 15, e0237912 (2020).

32. V. Sharma, D. M. Levi, S. A. Klein, Undercounting features and missing features: evidence for a high-level deficit in strabismic amblyopia. Nat Neurosci 3, 496-501 (2000).

33. O. Joly, E. Franko, Neuroimaging of amblyopia and binocular vision: a review. Front Integr Neurosci 8, 62-62 (2014).

34. C. Hou, Y. J. Kim, X. J. Lai, P. Verghese, Degraded attentional modulation of cortical neural populations in strabismic amblyopia. J Vis 16, 1-16 (2016).

35. M. Mortazavi, K. M. Aigner, J. E. Antono, C. Gambacorta, M. Nahum, D. M. Levi, J. Föcker, Neural correlates of visual spatial selective attention are altered at early and late processing stages in human amblyopia. Eur J Neurosci 53, 1086-1106 (2020). 
36. D. M. Levi, Linking assumptions in amblyopia. Vis Neurosci 30, 277-287 (2013).

37. M. Carrasco, C. Penpeci-Talgar, M. Eckstein, Spatial covert attention increases contrast sensitivity across the CSF: support for signal enhancement. Vision Res 40, 1203-1215 (2000).

38. Z.-L. Lu, B. A. Dosher, Spatial attention: Different mechanisms for central and peripheral temporal precues? J Exp Psychol Hum Percept Perform 26, 1534 (2000).

39. T. Liu, F. Pestilli, M. Carrasco, Transient attention enhances perceptual performance and FMRI response in human visual cortex. Neuron 45, 469-477 (2005).

40. F. Pestilli, S. Ling, M. Carrasco, A population-coding model of attention's influence on contrast response: Estimating neural effects from psychophysical data. Vision Res 49, 1144-1153 (2009).

41. K. Herrmann, L. Montaser-Kouhsari, M. Carrasco, D. J. Heeger, When size matters: attention affects performance by contrast or response gain. Nat Neurosci 13, 1554-1559 (2010).

42. Z.-L. Lu, X. Li, B. S. Tjan, B. A. Dosher, W. Chu, Attention extracts signal in external noise: A BOLD fMRI study. J Cogn Neurosci 23, 1148-1159 (2011).

43. L. Dugué, M. Roberts, M. Carrasco, Attention reorients periodically. Curr Biol 26, 15951601 (2016).

44. L. Dugué, E.P. Merriam, D.J. Heeger, M. Carrasco, Differential impact of endogenous and exogenous attention on activity in human visual cortex. Sci Rep 10, 1-16 (2020).

45. A. Fernández, M. Carrasco, Extinguishing exogenous attention via transcranial magnetic stimulation. Curr Biol 30, 4078-4084 (2020).

46. M. Jigo, M. Carrasco, Differential impact of exogenous and endogenous attention on the contrast sensitivity function across eccentricity. J Vis 20, 11-11 (2020).

47. M. A. Grubb et al., Exogenous spatial attention: evidence for intact functioning in adults with autism spectrum disorder. J Vis 13, 9-9 (2013).

48. M. A. Grubb et al., Endogenous spatial attention: evidence for intact functioning in adults with autism. Autism Res 6, 108-118 (2013).

49. M. Roberts, B. K. Ashinoff, F. X. Castellanos, M. Carrasco, When attention is intact in adults with ADHD. Psychon Bull Rev 25, 1423-1434 (2017).

50. G. S. Brown, K. G. White, The optimal correction for estimating extreme discriminability. Behav Res Methods 37, 436-449 (2005).

51. F. Wang, M. Chen, Y. Yan, L. Zhaoping, W. Li, Modulation of neuronal responses by exogenous attention in macaque primary visual cortex. J Neurosci 35, 13419-13429 (2015). 
52. M. Carrasco, B. McElree, Covert attention accelerates the rate of visual information processing. Proc Natl Acad Sci USA 98, 5363-5367 (2001).

53. R. Morey, Confidence intervals from normalized data: A correction to Cousineau (2005). Tutor Quant Methods Psychol 4, 61-64 (2008).

54. P. Verghese, S. P. McKee, D. M. Levi, Attention deficits in amblyopia. Curr Opin Psychol 29, 199-204 (2019).

55. A. A. Schoups, G. A. Orban, Interocular transfer in perceptual learning of a pop-out discrimination task. Proc Natl Acad Sci USA 93, 7358-7362 (1996).

56. R. Sabesan, A. Barbot, G. Yoon, Enhanced neural function in highly aberrated eyes following perceptual learning with adaptive optics. Vision Res 132, 78-84 (2017).

57. R. Wang, J. Y. Zhang, S. A. Klein, D. M. Levi, C. Yu, Task relevancy and demand modulate double-training enabled transfer of perceptual learning. Vision Res 61, 33-38 (2012).

58. M. Ahissar, S. Hochstein, Task difficulty and the specificity of perceptual learning. Nature 387, 401-406 (1997).

59. A. R. Seitz, Perceptual learning. Curr Biol 27, R631-R636 (2017).

60. S. C. Hung, A. R. Seitz, Prolonged training at threshold promotes robust retinotopic specificity in perceptual learning. J Neurosci 34, 8423-8431 (2014).

61. H. Harris, M. Gliksberg, D. Sagi, Generalized perceptual learning in the absence of sensory adaptation. Curr Biol 22, 1813-1817 (2012).

62. P. E. Jeter, B. A. Dosher, A. Petrov, Z. L. Lu, Task precision at transfer determines specificity of perceptual learning. J Vis 9, 1 1-13 (2009).

63. P. E. Jeter, B. A. Dosher, S. H. Liu, Z. L. Lu, Specificity of perceptual learning increases with increased training. Vision Res 50, 1928-1940 (2010).

64. A. Yashar, R. N. Denison, Feature reliability determines specificity and transfer of perceptual learning in orientation search. PLoS Comput Biol 13, e1005882 (2017).

65. R. W. Li, S. A. Klein, D. M. Levi, Prolonged perceptual learning of positional acuity in adult amblyopia: perceptual template retuning dynamics. J Neurosci 28, 14223-14229 (2008).

66. Y. Yotsumoto et al., Location-specific cortical activation changes during sleep after training for perceptual learning. Curr Biol 19, 1278-1282 (2009).

67. O. Yehezkel, A. Sterkin, M. Lev, D. M. Levi, U. Polat, Gains following perceptual learning are closely linked to the initial visual acuity. Sci Rep 6, 25188 (2016).

68. D. M. Levi, Visual processing in amblyopia: human studies. Strabismus 14, 11-19 (2006).

69. S. P. McKee, D. M. Levi, J. A. Movshon, The pattern of visual deficits in amblyopia. J Vis 3, 380-405 (2003). 
70. L. Asper, D. Crewther, S. G. Crewther, Strabismic amblyopia. Part 1. Psychophysics. Clin Exp Optom 83, 49-58 (2000).

71. M. Rolfs, Microsaccades: small steps on a long way. Vision Res 49, 2415-2441 (2009).

72. M. Poletti, M. Rucci, M. Carrasco, Selective attention within the foveola, Nat Neurosci 20, 1413-1419 (2017).

73. J.-Y. Zhang, L.-J. Cong, S. A. Klein, D. M. Levi, C. Yu, Perceptual learning improves adult amblyopic vision through rule-based cognitive compensation. Investig Ophthalmol Vis Sci 55, 2020-2030 (2014).

74. M. Ahissar, M. Nahum, I. Nelken, S. Hochstein, Reverse hierarchies and sensory learning. Philos Trans R Soc Lond B Biol Sci 364, 285-299 (2009).

75. K. Jia et al., Recurrent processing drives perceptual plasticity. Curr Biol 30, 4177-4187 (2020).

76. U. Polat et al., Training the brain to overcome the effect of aging on the human eye. Sci Rep 2, 278-278 (2012).

77. M. R. Cavanaugh et al., Visual recovery in cortical blindness is limited by high internal noise. J Vis 15, 9-9 (2015).

78. M. R. Cavanaugh, A. Barbot, M. Carrasco, K. R. Huxlin, Feature-based attention potentiates recovery of fine direction discrimination in cortically blind patients. Neuropsychologia 128, 315-324 (2019).

79. I. L. Bailey, J. E. Lovie-Kitchin, Visual acuity testing. From the laboratory to the clinic. Vision Res 90, 2-9 (2013).

80. N. Prins, F. A. A. Kingdom. (2009). Palamedes: Matlab routines for analyzing psychophysical data [Computer software]. (2009).

81. P. E. Jeter, B. A. Dosher, S. H. Liu, Z. L. Lu, Specificity of perceptual learning increases with increased training. Vision Res 50, 1928-1940 (2010).

82. A. M. Giordano, B. McElree, M. Carrasco, On the automaticity and flexibility of covert attention: a speed-accuracy trade-off analysis. J Vis 9, 1-10 (2009).

83. F. Hou et al., Evaluating the performance of the quick CSF method in detecting contrast sensitivity function changes. J Vis 16, 18-18 (2016).

84. F. Hou et al., qCSF in clinical application: efficient characterization and classification of contrast sensitivity functions in amblyopia. Investig Ophthalmol Vis Sci 51, 5365-5377 (2010).

85. W. Jia et al., The effects of monocular training on binocular functions in anisometropic amblyopia. Vision Res 152, 74-83 (2018).

86. C. B. Huang, Y. Zhou, Z. L. Lu, Broad bandwidth of perceptual learning in the visual system of adults with anisometropic amblyopia. Proc Natl Acad Sci USA 105, 4068-4073 (2008). 
87. D. Pelli, J. Robson, A. Wilkins, The design of a new letter chart for measuring contrast sensitivity. Clin Vision Sci 2, 187-199 (1988).

88. D. G. Pelli et al., A clinical test for visual crowding. F1000Research 5, (2016).

89. D. Pelli, H. Yiltiz, What internal noise source limits peripheral vision? J Vis 17, 775-775 (2017).

90. S. Song, D. M. Levi, D. G. Pelli, A double dissociation of the acuity and crowding limits to letter identification, and the promise of improved visual screening. J Vis 14, 3-3 (2014).

91. S. Hariharan, D. M. Levi, S. A. Klein, "Crowding" in normal and amblyopic vision assessed with Gaussian and Gabor C's. Vision Res 45, 617-633 (2005).

92. F. Strappini, D. G. Pelli, E. Di Pace, M. Martelli, Agnosic vision is like peripheral vision, which is limited by crowding. Cortex 89, 135-155 (2017).

93. Z. Hussain, B. S. Webb, A. T. Astle, P. V. McGraw, Perceptual learning reduces crowding in amblyopia and in the normal periphery. J Neurosci 32, 474-480 (2012).

94. K. Vancleef et al., ASTEROID: a new clinical Stereotest on an autostereo 3D tablet. Transl Vis Sci Technol 8, 25-25 (2019).

95. R. F. Hess, B. Thompson, Amblyopia and the binocular approach to its therapy. Vision Res 114, 4-16 (2015). 